\title{
Current correlation functions, QCD sum rules and vector mesons in baryonic matter *
}

\author{
F. Klingl, N. Kaiser and W. Weise \\ Physik-Department \\ Technische Universität München \\ Institut für Theoretische Physik \\ D-85747 Garching, Germany
}

\begin{abstract}
Based on an effective Lagrangian which combines chiral SU(3) dynamics with vector meson dominance, we have developed a model for the forward vector mesonnucleon scattering amplitudes. We use this as an input to calculate the low energy part of the current-current correlation function in nuclear matter. Its spectrum enters directly in the "left hand side" of QCD sum rules. For the isovector channel we find a significant enhancement of the in-medium spectral density below the $\rho$ resonance while the $\rho$ meson mass itself changes only slightly. The situation is different in the isoscalar channel, where the mass and peak position of the $\omega$ meson move downward while its width increases less drastically than in the $\rho$ meson case. For the $\phi$ meson we find almost no mass shift; the width of the peak broadens moderately. We observe a remarkable degree of consistency with the operator product expansion of QCD sum rules in all three channels. We point out, however, that these results cannot simply be interpreted, as commonly done, in terms of a universal rescaling of vector meson masses in matter.
\end{abstract}

\section{Introduction}

At present there is a lively discussion about the behaviour of vector mesons in dense and hot hadronic matter. According to Brown and Rho [1], vector meson masses $m_{V}$ (in fact the masses of most hadrons with the exception of the pion) should drop in matter according to a simple (BR) scaling law: $m_{V}^{*} / m_{V}=f_{\pi}^{*} / f_{\pi}$, where $f_{\pi}$ is the pion decay constant and the asterix refers to in-medium quantities. A decreasing $f_{\pi}^{*}$ (more precisely; the $f_{\pi}^{*}$ related to the time component of the axial current) in matter indicates a tendency toward chiral restoration (the transition from the spontaneously broken Nambu-Goldstone to the unbroken Wigner-Weyl realization of chiral symmetry). Dropping vector meson masses would then be a signal for this transition, if BR-scaling holds. Other scaling laws

*Work supported in part by GSI and BMBF 
[2] using a bag model find a mass reduction of two third times that of the nucleon mass which leads to a similar shift as BR-scaling.

Several analyses of the $\rho$ and $\omega$ meson masses in matter using QCD sum rules [3, 4] seem to confirm these expectations. On the other hand, model calculations of the density dependent two-pion self-energy of the rho meson in nuclear matter [5, 6] suggest only marginal changes of the in-medium rho meson mass, but a strongly increased decay width instead. Additional BR scaling seems to be needed in order to match the hadronic models with the QCD sum rule analysis [7]. Phenomenologically, a dropping $\rho$ meson mass seems to help 8, 9] understanding the enhanced dilepton yields seen at masses below the rho meson resonance in the CERES [10] and HELIOS [11] experiments at CERN.

Few attempts have been made so far to perform a realistic calculation of the omega meson self-energy in matter. The influence of the density dependent effective nucleon mass in the particle-hole polarization coupled to the $\omega$ meson has been studied in ref. 12]. However, leading in-medium effects from the $\omega$-decay into the three-pion continuum in the presence of nucleons have not yet been investigated. One of the aims of this paper is to take such effects into account.

The $\phi$ meson as the third neutral member of the SU(3) vector meson octet is special in the sense that it is almost a pure strange quark-antiquark system. The large strange quark mass protects it from effects related to chiral restoration. Therefore the $\phi$ meson mass does not seem to be rescaled in matter as strongly as the $\rho$ or $\omega$ meson when analyzed using in-medium QCD sum rules.

The QCD sum rule approach, at any practical level so far, has used only a caricature of the true spectral functions, namely a parameterization in terms of a delta function at the vector meson pole accompanied by a step function continuum at higher energies. While such a parameterization turns out to be valid in vacuum (as we shall confirm), this is shown not to be justified for the in-medium spectra. In particular, the parameterization of the rho meson in matter as an isolated quasiparticle pole becomes unreliable with increasing baryon density, given the strong increase of its partial decay width into $\rho N \rightarrow \pi N, \pi \pi N$ etc. channels.

The purpose of this paper is to present a systematic calculation of current-current correlation functions in baryonic matter, based on an effective Lagrangian which combines chiral symmetry with vector meson dominance of the electromagnetic current. In the isovector channel we find a significant enhancement of the low mass part of the spectrum with increasing baryon density. On the other hand the $\rho$ meson mass changes at a much slower rate with density than naively expected. In the isoscalar channel the situation turns out to be different. The position of the $\omega$ meson pole moves downward with rising density. The $\omega$ width does not increase as strongly as for the isovector channel. To leading order in the density, these features can be translated into statements about the (complex) effective scattering lengths of $\rho$ and $\omega$ interacting with a nucleon. These will be discussed in detail. The $\phi$ meson-nucleon scattering amplitude is much smaller than the $\rho N$ and $\omega N$ amplitudes, with a slight increase of the width but almost no shift of the resonance. We show that the vector meson-nucleon scattering amplitudes derived from our hadronic effective Lagrangian lead to spectral densities which are remarkably consistent with the standard QCD sum rule analysis, without the need for additional BR scaling.

This paper is organized as follows. In section 2 we review properties of the current 
correlation functions in vacuum and give a brief account of the QCD sum rule approach. The current-current correlation function in baryonic matter will be developed in section 3. To leading order in the density this requires a detailed calculation of the vector mesonnucleon scattering amplitudes which will be described in section 4. The in-medium spectra and their comparison with QCD sum rules is presented in section 5, and the results will be discussed in section 6 .

\section{Current-current correlation functions in vacuum}

\section{$2.1 \quad$ Basics}

This is an introductory reminder of properties of the current-current (CC) correlation function in vacuum (for a review see e.g. ref. [13]). The vector mesons are seen as resonances in the current-current $(\mathrm{CC})$ correlation function,

$$
\Pi_{\mu \nu}(q)=i \int d^{4} x e^{i q \cdot x}\left\langle 0\left|\mathcal{T} j_{\mu}(x) j_{\nu}(0)\right| 0\right\rangle
$$

where $\mathcal{T}$ denotes the time-ordered product and $j_{\mu}$ is the electromagnetic current which can be decomposed as

$$
j_{\mu}=j_{\mu}^{(\rho)}+j_{\mu}^{(\omega)}+j_{\mu}^{(\phi)}
$$

into vector currents specified by their quark content:

$$
\begin{aligned}
j_{\mu}^{(\rho)} & =\frac{1}{2}\left(\bar{u} \gamma_{\mu} u-\bar{d} \gamma_{\mu} d\right), \\
j_{\mu}^{(\omega)} & =\frac{1}{6}\left(\bar{u} \gamma_{\mu} u+\bar{d} \gamma_{\mu} d\right), \\
j_{\mu}^{(\phi)} & =-\frac{1}{3}\left(\bar{s} \gamma_{\mu} s\right) .
\end{aligned}
$$

Current conservation implies the following transverse tensor structure:

$$
\Pi_{\mu \nu}(q)=\left(g_{\mu \nu}-\frac{q_{\mu} q_{\nu}}{q^{2}}\right) \Pi\left(q^{2}\right),
$$

with the scalar CC correlation function

$$
\Pi\left(q^{2}\right)=\frac{1}{3} g^{\mu \nu} \Pi_{\mu \nu}(q) .
$$

Its imaginary part is proportional to the cross section for $e^{+} e^{-} \rightarrow$ hadrons:

$$
R(s)=\frac{\sigma\left(e^{+} e^{-} \rightarrow \text { hadrons }\right)}{\sigma\left(e^{+} e^{-} \rightarrow \mu^{+} \mu^{-}\right)}=-\frac{12 \pi}{s} \operatorname{Im} \Pi(s),
$$

where $\sqrt{s}$ is the total c.m. energy of the lepton pair and $\sigma\left(e^{+} e^{-} \rightarrow \mu^{+} \mu^{-}\right)=4 \pi \alpha^{2} / 3 s$ with $\alpha=e^{2} / 4 \pi=1 / 137$. The vector mesons can be distinguished by looking at different hadronic channels carrying the respective flavour (isospin) quantum numbers (see eqs.(3) 5)). The corresponding correlation functions are denoted by $\Pi^{(I=0)}, \Pi^{(I=1)}$ and $\Pi^{(\phi)}$. 
G-parity demands that the isovector current involving the $\rho$ meson can only couple to even numbers of pions (see the data in fig. 1a). Similarly, the annihilation of $e^{+} e^{-}$into odd numbers of pions determines the isoscalar current involving the $\omega$ meson, as can be seen in Figure 1b. Isospin violating processes are small but visible, such as the $\rho \omega$ mixing seen in the pion formfactor. The situation for the $\phi$ meson is more involved. The OZI rule would prohibit decays into pions. However, a violation of this rule by about five percent is observed in the three-pion channel. (Some experimental data near the $\phi$ resonance have been left out in Fig. 1b to emphasize the $\omega$-contribution given by the solid line). The $\phi$ meson decays mainly into OZI allowed channels such as $K^{+} K^{-}$or $K_{L} K_{S}$ (see Fig. 1c), but these channels also couple to the $\rho$ and $\omega$ mesons. The measured cross section of the annihilation into kaons includes an interference between all three vector mesons. (For more details see ref. [14]). Nevertheless the $\phi$ meson still dominates the data in the $e^{+} e^{-} \rightarrow K \bar{K}$ channels.

\subsection{Vector Meson Dominance update}

In the region $\sqrt{s} \lesssim 1 \mathrm{GeV}$ vector meson dominance (VMD) describes the experimental data of $e^{+} e^{-}$annihilation into hadronic channels very well. We briefly summarize here the results of ref. [14 based on an effective Lagrangian which combines chiral dynamics and vector meson dominance. This approach starts out from a "bare", stable vector meson with a mass $\stackrel{\circ}{m}_{V}$ slightly larger than the physical mass. Its propagator is $\stackrel{\circ}{D}_{V}\left(q^{2}\right)=$ $\left(q^{2}-\stackrel{\circ}{m}_{V}^{2}\right)^{-1}$. The coupling to the pseudoscalar mesons turns $\stackrel{\circ}{D} \underset{V}{ }$ into the physical vector meson propagator in vacuum,

$$
\begin{aligned}
D_{V}\left(q^{2}\right) & =\stackrel{\circ}{D}{ }_{V}\left(q^{2}\right)+\stackrel{\circ}{D}{ }_{V}\left(q^{2}\right) \Pi_{V}\left(q^{2}\right) D_{V}\left(q^{2}\right) \\
& =\frac{1}{q^{2}-\stackrel{\circ}{m}_{V}^{2}-\Pi_{V}\left(q^{2}\right)},
\end{aligned}
$$

with the complex vector meson self energy $\Pi_{V}$. The decay width of a given vector meson is related to the imaginary part of $\Pi_{V}$ by

$$
m_{V} \Gamma_{V}=-\operatorname{Im} \Pi_{V}\left(q^{2}=m_{V}^{2}\right),
$$

where $m_{V}^{2}=\stackrel{\circ}{m}_{V}^{2}+\operatorname{Re} \Pi_{V}\left(q^{2}=m_{V}^{2}\right)$ represents the squared physical vector meson mass.

\subsubsection{The $\rho$ meson}

For example, the coupling of the $\rho$ meson to the two-pion continuum leads to

$$
\operatorname{Im} \Pi_{\rho}\left(q^{2}\right)=-\frac{g^{2}}{48 \pi} q^{2}\left(1-\frac{4 m_{\pi}^{2}}{q^{2}}\right)^{\frac{3}{2}} \Theta\left(q^{2}-4 m_{\pi}^{2}\right),
$$

where $g=6.05$ is the $\rho \pi \pi$ coupling constant and $m_{\pi}=139.6 \mathrm{MeV}$ the charged pion mass. The real part of $\Pi_{\rho}$ can be derived from a dispersion relation. It has the form

$$
\operatorname{Re} \Pi_{\rho}\left(q^{2}\right)=c q^{2}-\frac{g^{2}}{24 \pi^{2}}\left[q^{2} \mathcal{G}\left(q^{2}, m_{\pi}^{2}\right)-4 m_{\pi}^{2}\right]
$$


with the function $\mathcal{G}\left(q^{2}, m^{2}\right)$ given explicitly in ref. 14]. The subtraction constant $c=0.16$ is determined by a fit to the isovector p-wave $\pi \pi$ scattering phase shift, which also fixes the bare rho meson mass $\stackrel{\circ}{m}_{\rho}=0.81 \mathrm{GeV}$. Note that in this approach the physical rho mass in vacuum, $m_{\rho}=0.77 \mathrm{MeV}$, is already shifted downward from the "bare" mass $\stackrel{\circ}{m}_{V}$. One may think of this "bare" rho meson as being a quark-antiquark bound state before turning on its strong coupling to the $\pi \pi$ continuum. ( In the $1 / N_{c}$ counting scheme, the bare rho meson mass is leading order in $N_{c}$, whereas the coupling to the $\pi \pi$ continuum via the complex self-energy $\Pi_{V}$ introduces $1 / N_{c}$ corrections.)

\subsubsection{The $\omega$ meson}

The relation (10) can be used to determine the imaginary part of the omega meson self energy by the decay into three pions $\left(\pi^{+}, \pi^{-}, \pi^{0}\right)$, as follows:

$$
\operatorname{Im} \Pi_{\omega}\left(q^{2}\right)=-\frac{q^{2}}{192 \pi^{3}} \iint d E_{+} d E_{-}\left[\vec{p}_{-}^{2} \vec{p}_{+}^{2}-\left(\vec{p}_{-} \cdot \vec{p}_{+}\right)^{2}\right]\left|F_{3 \pi}\right|^{2} .
$$

Here $E_{ \pm}$and $\vec{p}_{ \pm}$are the energies and momenta of the two charged pions in the final state, and the integration extends over the kinematically allowed region in the $\left(E_{+}, E_{-}\right)$plane. The amplitude $F_{3 \pi}$ represents the sum of the direct $\omega \rightarrow 3 \pi$ decay and the Gell-Mann, Sharp, Wagner (GSW) process in which the omega meson converts first into $\pi \rho$, followed by the decay of the $\rho$ meson into two pions:

$$
F_{3 \pi}=\frac{0.18}{f_{\pi}^{3}}+2.4 \frac{g}{f_{\pi}} \sum_{i} D_{\rho}\left(q-p_{i}\right) .
$$

Here we have introduced the pion decay constant $f_{\pi}=92.4 \mathrm{MeV}$, and $D_{\rho}$ is the rho meson propagator. The four-momentum $p_{i}$ refers to that of the first pion produced in the primary $\omega \rightarrow \pi \rho$ transition of the GSW process, and the index $i$ denotes the three possible charges of that primary pion. The determination of the coupling constants in eq.(14) is described in detail in ref.[14]. The real part of $\Pi_{\omega}$ can in principle be obtained by a dispersion relation. However, we choose to absorb this real part into the physical $\omega$ meson mass. This is justified since the momentum dependence of $\operatorname{Re} \Pi_{\omega}$ does not influence the results in any significant way [14], given the small omega meson width.

\subsubsection{The $\phi$ meson}

The self energy of the $\phi$ meson consists of three parts:

$$
\Pi_{\phi}=\Pi_{\phi \rightarrow K^{+} K^{-}}+\Pi_{\phi \rightarrow K_{L}^{0} K_{S}^{0}}+\Pi_{\phi \rightarrow 3 \pi} .
$$

The last term violates the OZI rule, but despite the small $\phi \omega$ mixing angle $\theta \simeq 0.05$ it contributes about fifteen percent of the total $\phi$ decay width. It is given by eq.(13) multiplied by the squared $\phi \omega$ mixing angle. The self energy parts coming from the decays into $K \bar{K}$ channels are of the same form as the $\pi \pi$ self energy of the $\rho$-meson and connected with the latter through $\mathrm{SU}(3)$ symmetry:

$$
\operatorname{Re} \Pi_{\phi \rightarrow K^{+} K^{-}}\left(q^{2}\right)=c_{K} q^{2}-\frac{g^{2}}{48 \pi^{2}}\left[q^{2} \mathcal{G}\left(q^{2}, m_{K^{+}}^{2}\right)-4 m_{K^{+}}^{2}\right],
$$




$$
\operatorname{Im} \Pi_{\phi \rightarrow K^{+} K^{-}}\left(q^{2}\right)=-\frac{g^{2}}{96 \pi} q^{2}\left(1-\frac{4 m_{K^{+}}^{2}}{q^{2}}\right)^{\frac{3}{2}} \Theta\left(q^{2}-4 m_{K^{+}}^{2}\right)
$$

For $\Pi_{\phi \rightarrow K_{L}^{0} K_{S}^{0}}$ the same expressions hold with the charged kaon mass $m_{K^{+}}$replaced by $m_{K^{0}}$. The subtraction constant $c_{K}=0.11$ has been determined in Ref. [14. Comparing the theoretical widths with empirical ones we find that $\mathrm{SU}(3)$ symmetry works well and gives the same universal coupling constant $g$ within ten percent [14] for all couplings of the vector and pseudoscalar meson octets.

\subsubsection{Current Correlation functions and VMD}

We can now establish the relation between the reduced electromagnetic CC correlation function $\Pi$ and the vector meson self-energies $\Pi_{V}$. Our starting point is again the effective Lagrangian of the coupled system of pseudoscalar mesons, vector mesons and photons as described in ref. [14]. This approach includes not only a direct conversion of a photon into a vector meson proportional to $e q^{2} / \stackrel{\circ}{g}_{V}$, but also a term which involves the photon interaction with pseudoscalar meson currents. The couplings $\stackrel{\circ}{g}_{V}$ are related through $\mathrm{SU}(3)$ symmetry as $\stackrel{\circ}{g}_{\rho}: \stackrel{o}{g}_{\omega}: \stackrel{o}{g}_{\phi}=1: 3: \frac{-3}{\sqrt{2}}$. The same relation holds for $g_{V}$, the strong couplings, where we identify $g_{\rho} \equiv g$. The result for $\Pi\left(q^{2}\right)$, expressed in terms of $\Pi_{V}\left(q^{2}\right)$, when iterating the vector meson self-energies to all orders is as follows:

$$
\Pi\left(q^{2}\right)=\sum_{V} \frac{1}{g_{V}^{2}}\left(\Pi_{V}\left(q^{2}\right)+\frac{\gamma_{V}^{2}\left(q^{2}\right) q^{4}}{q^{2}-\stackrel{\circ}{m}_{V}^{2}-\Pi_{V}\left(q^{2}\right)}\right) .
$$

This can easily be verified by direct diagrammatic analysis (see Fig. 2) using the framework of ref. [14]. Here $\gamma_{V}\left(q^{2}\right)$ represents the photon - vector meson vertex with inclusion of vertex corrections due to pion or kaon loops:

$$
\gamma_{V}\left(q^{2}\right)=a_{V}-\frac{\Pi_{V}\left(q^{2}\right)}{q^{2}}
$$

In case of the $\rho$ meson we have $a_{\rho}=g / \stackrel{\circ}{g}_{\rho} \simeq 1.1$, the ratio of the $\rho \pi \pi$ and $\rho \gamma$ couplings. In the limiting case of "complete" VMD where all of the photon-hadron interaction is transmitted via vector mesons, we have $a_{V} \equiv 1$. In the $\omega$ and $\phi$ channels the information about the direct $\gamma \leftrightarrow 3 \pi$ vertex is very limited and we therefore assume $a_{\omega}=a_{\phi}=1$. This gives an excellent description of the $e^{+} e^{-} \rightarrow \pi^{+} \pi^{0} \pi^{-}$cross section [14].

The imaginary part of $\Pi$ can now be easily evaluated:

$$
\operatorname{Im} \Pi\left(q^{2}\right)=\sum_{V} \frac{\operatorname{Im}_{V}\left(q^{2}\right)}{g_{V}^{2}}\left|F_{V}\left(q^{2}\right)\right|^{2},
$$

where

$$
F_{V}\left(q^{2}\right)=\frac{\left(1-a_{V}\right) q^{2}-\stackrel{\circ}{m}_{V}^{2}}{q^{2}-\stackrel{\circ}{m}_{V}^{2}-\Pi_{V}\left(q^{2}\right)}
$$


For example, in case of the rho meson, $F_{\rho}\left(q^{2}\right)$ is simply identified with the pion form factor:

$$
F_{\pi}\left(q^{2}\right)=\frac{\left(1-\frac{g}{\mathrm{o}_{\rho}}\right) q^{2}-\stackrel{\mathrm{o}}{m}_{\rho}^{2}}{q^{2}-\stackrel{\mathrm{o}}{m}_{\rho}^{2}-\Pi_{\rho}\left(q^{2}\right)}
$$

where again $g\left(\stackrel{o}{g}_{\rho}\right)$ is the coupling of the $\rho$ meson to the pion (photon) and $\Pi_{\rho}$ is the $\rho$ meson self energy specified in eqs.(11,12).

\subsubsection{High energy limit}

In the high energy region the measured correlation function approaches the asymptotic plateau predicted by perturbative QCD:

$$
R(s)=-\frac{12 \pi}{s} \operatorname{Im} \Pi(s)=\sum_{V} d_{V}\left(1+\frac{\alpha_{S}}{\pi}\right) \Theta\left(s-s_{V}\right)
$$

for $s$ larger than a characteristic scale $s_{V}$, where $d_{\rho}=3 / 2, d_{\omega}=1 / 6$ and $d_{\phi}=1 / 3$. This behaviour is clearly seen in the $\rho$-meson channel where the low mass (two-pion) region of the spectral function is evidently given by $\rho$ meson dominance in the form of eqs. (20) and (22). For $s>s_{\rho} \simeq 1.5 \mathrm{GeV}^{2}$ contributions from 4 and 6 pions take over and reach the asymptotic plateau above $s \sim 2 \mathrm{GeV}^{2}$.

\subsection{QCD sum rule analysis}

The basic idea of QCD sum rules is to match two representations of $\Pi\left(q^{2}\right)$ in the region of large spacelike $Q^{2}=-q^{2} \gg 1 \mathrm{GeV}$ for each one of the channels $V=\rho, \omega, \phi$ (we drop the channel index and always refer to a specific channel in this section). One representation is in terms of a twice subtracted dispersion relation:

$$
\Pi\left(q^{2}\right)=\Pi(0)+c q^{2}+\frac{q^{4}}{\pi} \int d s \frac{\operatorname{Im} \Pi(s)}{s^{2}\left(s-q^{2}-i \epsilon\right)},
$$

where the vanishing photon mass in the vacuum requires $\Pi(0)=0$. The second representation is in terms of the operator product expansion (OPE):

$$
\frac{12 \pi}{Q^{2}} \Pi\left(q^{2}=-Q^{2}\right)=\frac{d}{\pi}\left[-c_{0} \ln \left(\frac{Q^{2}}{\mu^{2}}\right)+\frac{c_{1}}{Q^{2}}+\frac{c_{2}}{Q^{4}}+\frac{c_{3}}{Q^{6}}+\ldots\right]
$$

(at a scale $\mu$ which is commonly chosen around $1 \mathrm{GeV}$ ). Here the coefficients $c_{1,2,3}$ incorporate the non-perturbative parts coming from the QCD condensates. In the isovector channel one finds

$$
\begin{aligned}
c_{0}^{\rho}= & 1+\frac{\alpha_{S}\left(Q^{2}\right)}{\pi}, \quad c_{1}^{\rho}=-3\left(m_{u}^{2}+m_{d}^{2}\right), \\
c_{2}^{\rho}= & \frac{\pi^{2}}{3}\left\langle 0\left|\frac{\alpha_{S}}{\pi} \mathcal{G}^{\mu \nu} \mathcal{G}_{\mu \nu}\right| 0\right\rangle+4 \pi^{2}\left\langle 0\left|m_{u} u \bar{u}+m_{d} d \bar{d}\right| 0\right\rangle, \\
c_{3}^{\rho}= & -4 \pi^{3}\left[\left\langle 0\left|\alpha_{S}\left(\bar{u} \gamma_{\mu} \gamma_{5} \lambda^{a} u-\bar{d} \gamma_{\mu} \gamma_{5} \lambda^{a} d\right)^{2}\right| 0\right\rangle\right. \\
& \left.+\frac{2}{9}\left\langle 0\left|\alpha_{S}\left(\bar{u} \gamma_{\mu} \lambda^{a} u+\bar{d} \gamma_{\mu} \lambda^{a} d\right) \sum_{q=u, d, s} \bar{q} \gamma^{\mu} \lambda^{a} q\right| 0\right\rangle\right] .
\end{aligned}
$$


Here $\alpha_{S}=4 \pi /\left(b \ln \left(Q^{2} / \Lambda_{Q C D}^{2}\right)\right)$ is the running coupling constant (with $\Lambda_{Q C D}=140 \mathrm{MeV}$ and $\left.b=11-(2 / 3) N_{f}=9\right)$. We can set $c_{1}$ equal to zero because its contribution is proportional to the squared mass of the light quarks and therefore negligible. For the isoscalar channel there is only a sign change between the up and down quark condensates of the first term in coefficient $c_{3}$.

The situation is different in the $\phi$ meson channel because of the large strange quark mass $m_{s}$. The coefficient $c_{1}^{\phi}$ is not negligible any longer and $c_{2}^{\phi}$ is larger than $c_{2}^{\rho, \omega}$. One finds 17

$$
\begin{aligned}
c_{0}^{\phi}= & 1+\frac{\alpha_{S}\left(Q^{2}\right)}{\pi}, \quad c_{1}^{\phi}=-6 m_{s}^{2}, \\
c_{2}^{\phi}= & \frac{\pi^{2}}{3}\left\langle 0\left|\frac{\alpha_{S}}{\pi} \mathcal{G}^{\mu \nu} \mathcal{G}_{\mu \nu}\right| 0\right\rangle+8 \pi^{2}\left\langle 0\left|m_{s} s \bar{s}\right| 0\right\rangle, \\
c_{3}^{\phi}= & -8 \pi^{3}\left[\left\langle 0\left|\alpha_{S}\left(\bar{s} \gamma_{\mu} \gamma_{5} \lambda^{a} s\right)^{2}\right| 0\right\rangle\right. \\
& \left.+\frac{4}{9}\left\langle 0\left|\alpha_{S}\left(\bar{s} \gamma_{\mu} \lambda^{a} s\right) \sum_{q=u, d, s} \bar{q} \gamma^{\mu} \lambda^{a} q\right| 0\right\rangle\right] .
\end{aligned}
$$

For the gluon condensate we use

$$
\left\langle 0\left|\frac{\alpha_{S}}{\pi} \mathcal{G}^{2}\right| 0\right\rangle=1.2 \cdot 10^{-2} \mathrm{GeV}^{4},
$$

the value determined from charmonium sum rules [17]. The four-quark condensates which contribute to $c_{3}$ are usually factorized by assuming that vacuum saturation holds, i.e. that they are proportional to $\langle 0|\bar{q} q| 0\rangle^{2}$. Then the coefficient $c_{3}$ is the same for all three channels. While this is a valid approximation in the large $N_{c}$ limit [18], possible deviations from vacuum saturation must be taken into account. We introduce a parameter $\kappa>1$ for this purpose and write:

$$
c_{3}=-\frac{896}{81} \kappa \pi^{3} \alpha_{S}\langle 0|\bar{q} q| 0\rangle^{2} .
$$

Here it is still assumed that the four-quark condensates with any two different flavours vanish. While this is not rigorously correct, the error can be estimated from $\rho \omega$ mixing 177. Its smallness suggests that this assumption may be appropriate. With the commonly accepted values $\langle 0|\bar{u} u| 0\rangle \simeq\langle 0|\bar{d} d| 0\rangle \simeq\langle 0|\bar{s} s| 0\rangle \equiv\langle 0|\bar{q} q| 0\rangle \simeq(-250 \mathrm{MeV})^{3}$ one needs $\kappa=2.36$ in order to obtain the same value of $c_{3}$ as originally proposed by Shifman et al. [17]. Note that Hatsuda et al. [19] use a larger value $\langle 0|\bar{q} q| 0\rangle=(-280 \mathrm{MeV})^{3}$ in their approach. We also mention here that strongly increased values of $c_{3}$ as suggested in refs. [20, 21] would not be compatible with our analysis.

The QCD sum rule approach compares the dispersion relation of eq.(24) on the left side with its OPE representation (eq.(25), right side). We use our calculated

$$
R^{(V)}(s)=-\frac{12 \pi}{s g_{V}^{2}} \operatorname{Im} \Pi_{V}(s)\left|F_{V}(s)\right|^{2} \Theta\left(s_{V}-s\right)+d_{V}\left(1+\frac{\alpha_{S}}{\pi}\right) \Theta\left(s-s_{V}\right)
$$

to determine the left side. Since the OPE on the right side of eq.(25) is truncated at order $Q^{-6}$ we apply the usual Borel transformation [16, 17] in order to improve convergence:

$$
\hat{f}\left(\mathcal{M}^{2}\right)=\lim _{\substack{Q^{2} \rightarrow \infty, n \rightarrow \infty,\left(Q^{2} / n \equiv \mathcal{M}^{2}=\text { const. }\right)}} \frac{1}{(n-1) !}\left(Q^{2}\right)^{n}\left(-\frac{d}{d Q^{2}}\right)^{n} f\left(Q^{2}\right) .
$$


After Borelization the QCD sum rule for each $R^{(V)}$ becomes

$$
\frac{1}{\mathcal{M}^{2} d}\left[12 \pi^{2} \Pi(0)+\int_{0}^{\infty} d s R(s) e^{-s / \mathcal{M}^{2}}\right]=c_{0}+\frac{c_{1}}{\mathcal{M}^{2}}+\frac{c_{2}}{\mathcal{M}^{4}}+\frac{c_{3}}{2 \mathcal{M}^{6}}
$$

Given $R^{(V)}(s)$ of eq. 34) this represents a consistency condition which should hold independently of the Borel mass parameter $\mathcal{M}$ within the range of applicability of the QCD sum rules, i.e. for $\mathcal{M}$ larger than a certain minimal value. Note again in the vacuum $\Pi(0)$ vanishes, but we have kept it here since it will not vanish in matter.

In Fig. 3 we compare the "left side" of eq.(36) calculated from eq.(34) with the "right side" as a function of the Borel mass $\mathcal{M}$. There is excellent agreement in all three channels between both sides of the sum rule, down to $\mathcal{M} \simeq 0.8 \mathrm{GeV}$ where the convergence of the OPE series (25) starts to break down and higher order terms become important. We have checked that our condensates agree with the "optimal" values, using the fit program MINUIT. It determines the condensate parameters to be

$$
\begin{aligned}
c_{2}^{\rho, \omega} & =0.04 \mathrm{GeV}^{4}, \\
c_{3}^{\rho, \omega} & =-0.071 \mathrm{GeV}^{6}
\end{aligned}
$$

together with $s_{\rho, \omega}=1.56 \mathrm{GeV}^{2}$ and

$$
\begin{aligned}
c_{1}^{\phi} & =0.07 \mathrm{GeV}^{2}, \\
c_{2}^{\phi} & =-0.10 \mathrm{GeV}^{4}, \\
c_{3}^{\phi} & =-0.07 \mathrm{GeV}^{6},
\end{aligned}
$$

taking $s_{\phi}=2.2 \mathrm{GeV}^{2}$.

We also confirm previous results [17] which use the simplified ansatz for the spectra,

$$
R^{(V)}=\mathcal{F}_{V} \delta\left(s-m_{V}^{2}\right)+d_{V}\left(1+\frac{\alpha_{S}}{\pi}\right) \Theta\left(s-s_{V}\right)
$$

with $\mathcal{F}_{\rho}=9 \mathcal{F}_{\omega}=2.4, m_{\rho, \omega}=0.77 \mathrm{GeV}, s_{\rho, \omega}=1.5 \mathrm{GeV}^{2}$ and $\mathcal{F}_{\phi}=0.79, m_{\phi}=1.02 \mathrm{GeV}$, $s_{\phi}=2.2 \mathrm{GeV}^{2}$. Including the vector meson decay widths does not make a substantial difference, at least not in vacuum: after taking the Borel transform their effects are suppressed as $\left(m_{V} \Gamma_{V} / \mathcal{M}^{2}\right)^{2}$. This explains why the large difference of the $\rho$ and $\omega$ meson decay widths does not affect the OPE. However, for fine tuning purposes it is essential to use the complete spectrum (34). For the in-medium sum rule the use of a realistic spectrum is mandatory as discussed in section 5. We also note that the approximate

degeneracy of the $\rho$ and $\omega$ meson masses emerges naturally in the OPE. This is one of the traditional successes of QCD sum rules.

\section{Correlation functions in baryonic matter}

We now replace the vacuum $|0\rangle$ by a state $|\Omega(\rho)\rangle$ of matter with given baryon density $\rho$. The CC correlation function to leading order in $\rho$ has been written down before [19, 22]. In this chapter we briefly summarize the steps which lead to this first order term, and 
we also describe its iteration to all orders in density. The basic input into this scheme is the forward Compton amplitude for scattering of a timelike, virtual photon on the nucleon. Our task is to calculate this amplitude using an effective Lagrangian approach. The resulting spectral function in matter will then be further analyzed using a QCD sum rule with in-medium condensates.

\subsection{Basics}

In the present paper we restrict ourselves to the case of matter at temperature $T=0$. The matter may be moving with a constant four-velocity $v^{\mu}$. We choose a frame of reference in which matter as a whole is at rest by setting $\vec{v}=0$. The four-momentum $q^{\mu}=(\omega, \vec{q})$ that enters in the $\mathrm{CC}$ correlation function is from now on understood to be taken in this particular frame. The generalization of the $\mathrm{CC}$ correlation function to the case of baryonic matter is

$$
\Pi_{\mu \nu}(\omega, \vec{q} ; \rho)=i \int_{-\infty}^{+\infty} d t \int d^{3} x e^{i \omega t-i \vec{q} \cdot \vec{x}}\left\langle\Omega(\rho)\left|\mathcal{T} j_{\mu}(t, \vec{x}) j_{\nu}(0)\right| \Omega(\rho)\right\rangle .
$$

For the vacuum case $(\rho=0)$ we have seen that at squared virtual photon masses $q^{2}=$ $\omega^{2}-\vec{q}^{2} \lesssim 1 \mathrm{GeV}^{2}$, the spectrum of $\Pi$ starts at $q^{2}=4 m_{\pi}^{2}$ in the isovector channel, at $q^{2}=9 m_{\pi}^{2}$ in the isoscalar channel and at $q^{2}=4 m_{K}^{2}$ in the $\phi$ channel (apart from small $\phi \rightarrow 3 \pi$ contribution which also starts at $q^{2}=9 m_{\pi}^{2}$ ). This spectrum displays the vector mesons as the dominant doorway states which decay into pseudoscalar mesons. In matter we have new processes involving nucleons, such as

$$
\gamma^{*} N \rightarrow \pi N, \pi \pi N, K \Lambda, K \Sigma, \quad \text { etc. }
$$

which start at $q^{2} \geq m_{\pi}^{2}$ or at the threshold for kaon production. In addition there are nuclear particle-hole excitations at very low energies which need not be considered here.

For a nuclear Fermi gas with equal number of protons and neutrons, Fermi momentum

$p_{F}$ and density $\rho=2 p_{F}^{3} /\left(3 \pi^{2}\right)$, the density dependent matrix element $\langle\Omega(\rho)|\hat{\mathcal{O}}| \Omega(\rho)\rangle$ of any operator $\hat{\mathcal{O}}$ is given by [23]:

$$
\langle\Omega(\rho)|\hat{\mathcal{O}}| \Omega(\rho)\rangle=\langle 0|\hat{\mathcal{O}}| 0\rangle+4 \int_{|\vec{p}| \leq p_{F}} \frac{d^{3} p}{(2 \pi)^{3}} \frac{M}{E(\vec{p})}\langle N(\vec{p})|\hat{\mathcal{O}}| N(\vec{p})\rangle+\ldots,
$$

where $|N(\vec{p})\rangle$ is a proton or neutron state (including spin) with momentum $\vec{p}$ and the normalization

$$
\left\langle N(\vec{p}) \mid N\left(\vec{p}^{\prime}\right)\right\rangle=(2 \pi)^{3} \frac{E(\vec{p})}{M} \delta^{3}\left(\vec{p}-\vec{p}^{\prime}\right),
$$

with $E(\vec{p})=\sqrt{\vec{p}^{2}+M^{2}}$. The dots in eq. (45) indicate higher orders in density.

When applied to the CC correlation function in matter, eq.(45) becomes

$$
\Pi_{\mu \nu}(\omega, \vec{q} ; \rho)=\Pi_{\mu \nu}^{\mathrm{vac}}(\omega, \vec{q})+4 \int_{|\vec{p}| \leq p_{F}} \frac{d^{3} p}{(2 \pi)^{3}} \frac{M}{E(\vec{p})} T_{\mu \nu}(\omega, \vec{q} ; \vec{p})+\ldots,
$$


where the first term $\Pi_{\mu \nu}^{\mathrm{vac}}=\Pi_{\mu \nu}(\rho=0)$ is just the vacuum CC correlation tensor (11) discussed previously, and

$$
T_{\mu \nu}(\omega, \vec{q} ; \vec{p})=i \int_{-\infty}^{+\infty} d t \int d^{3} x e^{i \omega t-i \vec{q} \cdot \vec{x}}\left\langle N(\vec{p})\left|\mathcal{T} j_{\mu}(t, \vec{x}) j_{\nu}(0)\right| N(\vec{p})\right\rangle
$$

is the forward Compton scattering tensor for a virtual photon of energy $\omega$ and momentum $\vec{q}$ on a free nucleon. The tensor $T_{\mu \nu}$ has the standard decomposition

$$
T_{\mu \nu}=\left(\frac{q_{\mu} q_{\nu}}{q^{2}}-g_{\mu \nu}\right) T_{1}+\left(p_{\mu}-\frac{p \cdot q}{q^{2}} q_{\mu}\right)\left(p_{\nu}-\frac{p \cdot q}{q^{2}} q_{\nu}\right) \frac{T_{2}}{M^{2}},
$$

which defines the amplitudes $T_{1}$ and $T_{2}$. It is often more useful to introduce the amplitudes

$$
\begin{aligned}
& T^{(T)}=T_{1}, \\
& T^{(L)}=T_{1}-\left[1-\frac{(p \cdot q)^{2}}{p^{2} q^{2}}\right] T_{2},
\end{aligned}
$$

and reexpress $T_{\mu \nu}$ as

$$
T_{\mu \nu}=-\left(P_{L}\right)_{\mu \nu} T^{(L)}-\left(P_{T}\right)_{\mu \nu} T^{(T)},
$$

where the matrices $P_{L}$ and $P_{T}$ act as projectors on longitudinal and transverse parts with the properties: $P_{L}^{2}=P_{L}, P_{T}^{2}=P_{T}$ and $P_{L} P_{T}=P_{T} P_{L}=0$. For a nucleon at rest with $p^{\mu}=(M, \vec{p}=0)$ the tensors $P_{L}$ and $P_{T}$ are given by

$$
P_{L}=-\left[\begin{array}{cc}
\frac{\vec{q}^{2}}{q^{2}} & \frac{\omega q^{j}}{q^{2}} \\
\frac{\omega q^{i}}{q^{2}} & \frac{q^{i} q^{j} \omega^{2}}{q^{2} \vec{q}^{2}}
\end{array}\right], \quad P_{T}=-\left[\begin{array}{cc}
0 & 0 \\
0 & \delta^{i j}-\frac{q^{i} q^{j}}{\vec{q}^{2}}
\end{array}\right] .
$$

\subsection{Low density limit}

At low baryon density $\rho$ the $\vec{p}$ dependence of the Compton tensor in eq.(47) can be neglected and we have

$$
\begin{aligned}
\Pi_{\mu \nu}(\omega, \vec{q} ; \rho) & \simeq \Pi_{\mu \nu}^{\mathrm{vac}}(q)+\rho T_{\mu \nu}(\omega, \vec{q} ; \vec{p}=0)+\ldots \\
& =\Pi_{\mu \nu}^{\mathrm{vac}}(q)-\rho\left[\left(P_{L}\right)_{\mu \nu} T^{(L)}(\omega, \vec{q}, \vec{p}=0)+\left(P_{T}\right)_{\mu \nu} T^{(T)}(\omega, \vec{q}, \vec{p}=0)\right]+\ldots
\end{aligned}
$$

We introduce the notation $T_{\mu \nu}(\omega, \vec{q}) \equiv T_{\mu \nu}(\omega, \vec{q} ; \vec{p}=0)$ etc. from here on. The reduced CC correlation function $\Pi(\omega, \vec{q} ; \rho)=g_{\mu \nu} \Pi^{\mu \nu} / 3$ analogous to eq.(7) becomes

$$
\begin{aligned}
\Pi(\omega, \vec{q} ; \rho) & =\Pi^{\mathrm{vac}}(q)-\rho\left(\frac{1}{3} T^{(L)}(\omega, \vec{q})+\frac{2}{3} T^{(T)}(\omega, \vec{q})\right) \\
& =\Pi^{\mathrm{vac}}(q)-\rho\left(T_{1}(\omega, \vec{q})+\frac{\vec{q}^{2}}{3 q^{2}} T_{2}(\omega, \vec{q})\right),
\end{aligned}
$$

to leading order in $\rho$. These expressions have a direct analogue when looking at the leading density dependence of the vector meson self-energy tensor:

$$
\Pi_{V}^{\mu \nu}(\omega, \vec{q} ; \rho)=\Pi_{V}^{\operatorname{vac} \mu \nu}\left(q^{2}\right)+\rho T_{V N}^{\mu \nu}(\omega, \vec{q})+\ldots
$$


where $\Pi_{V}^{\mathrm{vac} \mu \nu}=\Pi_{V}^{\mu \nu}(\rho=0)$ is the vacuum contribution and $T_{V N}^{\mu \nu}(\omega, \vec{q})$ denotes the (offshell) vector meson-nucleon amplitude analogous to the Compton tensor $T^{\mu \nu}$. With the help of the projectors $P_{T}$ and $P_{L}$ we can separate this self energy into two parts:

$$
\Pi_{V}^{\mu \nu}(\omega, \vec{q} ; \rho)=\left[\Pi_{V}^{\mathrm{vac}}\left(q^{2}\right)-\rho T_{V N}^{(L)}\right]\left(P_{L}\right)_{\mu \nu}+\left[\Pi_{V}^{\mathrm{vac}}\left(q^{2}\right)-\rho T_{V N}^{(T)}\right]\left(P_{T}\right)_{\mu \nu},
$$

where $\Pi_{V}^{\mathrm{vac}}$ is the scalar self-energy function in the vacuum and $T_{V N}^{(L, T)}$ are the longitudinal and transverse $V N$ scattering amplitudes analogous to the Compton amplitudes $T^{(L, T)}$ of eqs. (50,51).

\subsection{Vector meson propagator and current correlation function in medium}

Using the projectors $P_{L}$ and $P_{T}$ it is now a straightforward exercise to decompose the tensor structure of the in-medium vector meson propagator. We find

$$
D_{V}^{\mu \nu}(\omega, \vec{q} ; \rho)=\frac{\left(P_{L}\right)^{\mu \nu}}{q^{2}-\stackrel{\circ}{m}_{V}^{2}-\left(\Pi_{V}^{\operatorname{vac}}\left(q^{2}\right)-\rho T_{V N}^{(L)}\right)}+\frac{\left(P_{T}\right)^{\mu \nu}}{q^{2}-\stackrel{\circ}{m}_{V}^{2}-\left(\Pi_{V}^{\mathrm{vac}}\left(q^{2}\right)-\rho T_{V N}^{(T)}\right)} .
$$

The leading medium corrections, iterated to all orders, are therefore simply obtained by the replacement $\Pi_{V}^{\mathrm{vac}}\left(q^{2}\right) \rightarrow \Pi_{V}^{\mathrm{vac}}-\rho T_{V N}^{(L, T)}$ in the transverse and longitudinal parts, respectively.

The structure seen in eq. (58) translates directly into the in-medium CC correlation function (43). Using diagrammatic techniques analogous to those in the vacuum one derives:

$$
\Pi^{\mu \nu}(\omega, \vec{q} ; \rho)=\Pi^{(L)}(\omega, \vec{q} ; \rho)\left(P_{L}\right)^{\mu \nu}+\Pi^{(T)}(\omega, \vec{q} ; \rho)\left(P_{T}\right)^{\mu \nu},
$$

with the longitudinal and transverse parts

$$
\begin{aligned}
& \Pi^{(L, T)}(\omega, \vec{q} ; \rho)= \\
& \sum_{V} \frac{1}{g_{V}^{2}}\left(\Pi_{V}^{\mathrm{vac}}\left(q^{2}\right)-\rho T_{V N}^{(L, T)}(\omega, \vec{q})+\frac{\left[\gamma_{V}^{(L, T)}(w, \vec{q} ; \rho)\right]^{2} q^{4}}{q^{2}-\stackrel{\mathrm{o}}{m}_{V}^{2}-\Pi_{V}^{\mathrm{vac}}\left(q^{2}\right)+\rho T_{V N}^{(L, T)}(\omega, \vec{q})}\right)
\end{aligned}
$$

and

$$
\gamma_{V}^{(L, T)}(\omega, \vec{q} ; \rho)=a_{V}-\frac{\Pi_{V}^{\mathrm{vac}}-\rho T_{V N}^{(L, T)}(\omega, \vec{q})}{q^{2}} .
$$

This result generalizes eqs.(18, 19). The imaginary parts of $\Pi^{(L)}$ and $\Pi^{(T)}$ become:

$$
\operatorname{Im} \Pi^{(L, T)}=\sum_{V} \frac{1}{g_{V}^{2}} \operatorname{Im}\left(\Pi_{V}^{\mathrm{vac}}-\rho T_{V N}^{(L, T)}\right)\left|\frac{\left(1-a_{V}\right) q^{2}-\stackrel{\mathrm{o}}{m}_{V}^{2}}{q^{2}-\stackrel{\mathrm{o}}{m}_{V}^{2}-\Pi_{V}^{\mathrm{vac}}+\rho T_{V N}^{(L, T)}}\right|^{2} .
$$

This is the in-medium generalization of eq.(20), with $\Pi_{V}^{\mathrm{vac}}$ replaced everywhere by $\Pi_{V}^{\mathrm{vac}}-$ $\rho T_{V N}^{(L, T)}$. 
It is instructive to recover the low-density limit for which one finds:

$$
\Pi_{\mu \nu}(\omega, \vec{q} ; \rho)=\Pi_{\mu \nu}^{\mathrm{vac}}\left(q^{2}\right)-\rho \sum_{V} \frac{1}{g_{V}^{2}} F_{V}\left(q^{2}\right)\left[T_{V N}^{(L)}\left(P_{L}\right)_{\mu \nu}+T_{V N}^{(T)}\left(P_{T}\right)_{\mu \nu}\right] F_{V}\left(q^{2}\right)+\ldots
$$

where $F_{V}\left(q^{2}\right)$ are the form factors given in eq.(21). We can now identify the relationship between the Compton scattering amplitude and the $V N$ amplitude by comparison with eq.(54):

$$
T_{\mu \nu}=\sum_{V} \frac{1}{g_{V}^{2}} F_{V}\left[T_{V N}^{(L)}\left(P_{L}\right)_{\mu \nu}+T_{V N}^{(T)}\left(P_{T}\right)_{\mu \nu}\right] F_{V}
$$

or

$$
T^{(L, T)}=\sum_{V} \frac{F_{V}^{2}}{g_{V}^{2}} T_{V N}^{(L, T)}
$$

In the limiting case of a real photon with $q^{2}=\omega^{2}-\vec{q}^{2}=0$ we have $F_{V}\left(q^{2}=0\right)=1$ and find the simpler relation

$$
T^{(L, T)}(\omega=|\vec{q}|)=\sum_{V} \frac{T_{V N}^{(L, T)}(\omega=|\vec{q}|)}{g_{V}^{2}} .
$$

Furthermore, $\Pi^{\operatorname{vac}}\left(q^{2}=0\right)=0$ and $\operatorname{Im} T^{(L)}=0$ for real photons so that we obtain, to leading order in the density $\rho$ :

$$
\operatorname{Im}_{\mu \nu}(\omega=|\vec{q}| ; \rho)=-\rho \operatorname{Im} T^{(T)}(\omega=|\vec{q}|)\left(P_{T}\right)_{\mu \nu}
$$

and therefore

$$
\sum_{V} \frac{\operatorname{Im} T_{V N}^{(T)}(\omega=|\vec{q}|)}{g_{V}^{2}}=\frac{\omega}{4 \pi \alpha} \sigma_{\gamma N}(\omega)
$$

where $\sigma_{\gamma N}$ is the total photon-nucleon cross section averaged over proton and neutron and we have used the optical theorem

$$
\sigma_{\gamma N}(\omega)=e^{2} \operatorname{Im} T^{(T)}(\omega=|\vec{q}|) / \omega .
$$

\subsection{Vector mesons at rest}

From now on we focus on the simpler case $\vec{q}=0$ in which the vector mesons are at rest. Note that this is also the situation corresponding to back-to-back emission of an electron-positron pair. In this case we have

$$
T(\omega) \equiv-\frac{1}{3} g_{\mu \nu} T^{\mu \nu}(\omega, \vec{q}=0)=T_{1}(\omega, \vec{q}=0)=T^{(T)}(\omega, \vec{q}=0)=T^{(L)}(\omega, \vec{q}=0) .
$$

The reduced CC correlation function in the low density limit (eq.(54)) now becomes

$$
\Pi(\omega, \vec{q}=0 ; \rho)=\Pi^{\mathrm{vac}}\left(\omega^{2}\right)-\rho T(\omega)+\ldots,
$$

and for the vector meson self energy we find

$$
\Pi_{V}(\omega, \vec{q}=0 ; \rho)=\Pi_{V}^{\mathrm{vac}}\left(\omega^{2}\right)-\rho T_{V N}(\omega)+\ldots,
$$


where $T_{V N}=T_{V N}^{(L)}=T_{V N}^{(T)}$ denotes the (off-shell) vector meson-nucleon amplitude for $\vec{q}=0$, analogous to the Compton amplitude $T\left(\omega^{2}\right)$. The full in-medium propagator (58), for a vector meson at rest, simplifies to $D_{V}^{00}=D_{V}^{0 j}=D_{V}^{i 0}=0$ and

$$
D_{V}^{i j}(\omega, \vec{q}=0 ; \rho)=\frac{-\delta^{i j}}{\omega^{2}-\stackrel{\mathrm{o}}{m}_{V}^{2}-\left(\Pi_{V}^{\mathrm{vac}}\left(\omega^{2}\right)-\rho T_{V N}\right)}
$$

The reduced CC correlation function in matter which follows from eq.(59) is now:

$$
\Pi(\omega, \vec{q}=0, \rho)=\sum_{V} \frac{1}{g_{V}^{2}}\left(\Pi_{V}^{\mathrm{vac}}\left(\omega^{2}\right)-\rho T_{V N}+\frac{\gamma_{V}^{2}(\omega ; \rho) \omega^{4}}{\omega^{2}-\stackrel{\mathrm{o}}{m}_{V}^{2}-\Pi_{V}^{\mathrm{vac}}\left(\omega^{2}\right)+\rho T_{V N}}\right),
$$

with

$$
\gamma_{V}(\omega ; \rho)=a_{V}-\frac{\prod_{V}^{\mathrm{vac}}\left(\omega^{2}\right)-\rho T_{V N}(\omega)}{\omega^{2}}
$$

Its imaginary part enters directly in the back-to-back dilepton production rate and it is also the key input for the "left side" of the QCD sum rule:

$$
\begin{aligned}
& \operatorname{Im} \Pi(\omega, \vec{q}=0 ; \rho)= \\
& \sum_{V} \frac{1}{g_{V}^{2}} \operatorname{Im}\left(\Pi_{V}^{\mathrm{vac}}\left(\omega^{2}\right)-\rho T_{V N}(\omega)\right)\left|\frac{\left(1-a_{V}\right) \omega^{2}-\stackrel{\text { o }}{2}_{V}^{2}}{\omega^{2}-\stackrel{\circ}{m}_{V}^{2}-\Pi_{V}^{\mathrm{vac}}\left(\omega^{2}\right)+\rho T_{V N}(\omega)}\right|^{2} .
\end{aligned}
$$

We have thus established the relationship between the isovector $\mathrm{CC}$ correlation function in matter and the (complex) off-shell vector meson-nucleon amplitudes.

\section{$4 \quad$ Vector meson-nucleon scattering amplitudes}

\subsection{Effective Lagrangian}

In order to determine the low energy $V N$ scattering amplitudes, we use the hadronic effective Lagrangian of ref.[14] which combines chiral SU(3) dynamics with VMD. This approach has been successfully applied for vacuum processes. The extended Lagrangian incorporates nucleons, hyperons (and also $\Delta$ 's in intermediate states ) with pseudovector meson-baryon interactions:

$$
\mathcal{L}_{\Phi B}=F \operatorname{tr}\left(\bar{B} \gamma_{\mu} \gamma_{5}\left[u^{\mu}, B\right]\right)+D \operatorname{tr}\left(\bar{B} \gamma_{\mu} \gamma_{5}\left\{u^{\mu}, B\right\}_{+}\right)
$$

where $B(\Phi)$ represent the $\mathrm{SU}(3)$ matrix fields of the baryon (pseudoscalar meson) octet and

$$
u^{\mu}=-\frac{1}{2 f_{\pi}}\left(\partial^{\mu} \Phi-i e[Q, \Phi] A^{\mu}\right) .
$$

This effective Lagrangian has been tested successfully in the $\mathrm{SU}(3)$ coupled channels approach of refs. 24]. Here $Q$ denotes the quark charge matrix, $A^{\mu}$ is the photon field and $f_{\pi}=92.4 \mathrm{MeV}$ is the pion decay constant. The second part of $u^{\mu}$ gives rise to the Kroll-Ruderman couplings of the photon with the interacting system of baryons and 
pseudoscalar mesons. The parameters $F \simeq 0.51$ and $D \simeq 0.75$ satisfy the constraint $g_{A}=F+D=1.26$. The vector meson-baryon interactions are then introduced by the minimal coupling scheme which substitutes $e Q A^{\mu}$ by the vector field $g V^{\mu} / 2$, where

$$
V^{\mu} \equiv\left(\begin{array}{ccc}
\rho^{\mu}+\omega^{\mu} & 0 & 0 \\
0 & -\rho^{\mu}+\omega^{\mu} & 0 \\
0 & 0 & \sqrt{2} \phi^{\mu}
\end{array}\right) \text {. }
$$

In the Kroll-Ruderman part this leads to

$$
\mathcal{L}_{V \Phi B}=\frac{i g}{4 f_{\pi}}\left(F \operatorname{tr}\left(\bar{B} \gamma_{\mu} \gamma_{5}\left[\left[V^{\mu}, \Phi\right], B\right]\right)+D \operatorname{tr}\left(\bar{B} \gamma_{\mu} \gamma_{5}\left\{\left[V^{\mu}, \Phi\right], B\right\}_{+}\right)\right) .
$$

The direct coupling of the photon to the baryons becomes:

$$
\mathcal{L}_{\gamma B}=e\left(\operatorname{tr}\left(\bar{B} \gamma_{\mu}[Q, B]\right)-\operatorname{tr}\left(\bar{B} \gamma_{\mu} B\right) \operatorname{tr}(Q)\right) A^{\mu} .
$$

The second term vanishes, because the trace of the $u-, d-$ and $s$-quark charge matrix is zero. When determining the direct vector meson-baryon coupling by the replacement $e Q A^{\mu} \rightarrow g V^{\mu} / 2$ this term survives and we find:

$$
\mathcal{L}_{V B}^{(1)}=\frac{g}{2}\left(\operatorname{tr}\left(\bar{B} \gamma_{\mu}\left[V^{\mu}, B\right]\right)-\operatorname{tr}\left(\bar{B} \gamma_{\mu} B\right) \operatorname{tr}\left(V^{\mu}\right)\right) .
$$

This form implies the $\mathrm{SU}(3)$ symmetry relation $g \equiv g_{\rho N}=\frac{1}{3} g_{\omega N}=-\frac{\sqrt{2}}{3} g_{\phi N}$. We also include corrections due to anomalous $V N$ tensor couplings with $\kappa_{\rho}=6.0$ and $\kappa_{\omega}=0.1$ :

$$
\mathcal{L}_{V N}^{(2)}=\frac{g \kappa_{\rho}}{4 M_{N}} \bar{N} \vec{\tau} \sigma_{\mu \nu} N \partial^{\mu} \vec{\rho}^{\nu}+\frac{g \kappa_{\omega}}{4 M_{N}} \bar{N} \sigma_{\mu \nu} N \partial^{\mu} \omega^{\nu}
$$

In the strict $\mathrm{SU}(3)$ limit we set $\kappa_{\phi}=0$ and recognize that there is no direct $\phi$ mesonnucleon coupling. The $\phi N$ interaction in this channel arises entirely from kaon loops.

The interaction vertices obtained from the effective Lagrangian are summarized in Fig. 4. The minimal coupling scheme for the vector mesons makes sure that we correctly approach the limit $\omega \rightarrow 0$ keeping $\vec{q}=0$.

Using this effective Lagrangian we now calculate the $\rho, \omega$ and $\phi$ meson-nucleon scattering amplitudes $T_{V N}(\omega)$, with the vector mesons at rest $(\vec{q}=0)$. The imaginary parts of these scattering amplitudes are then evaluated using standard Cutkosky rules. The real parts are determined by a subtracted dispersion relation at $\vec{q}=0$ :

$$
\operatorname{Re} T_{V N}(\omega)=l_{V}+\frac{\omega^{2}}{\pi} \mathcal{P} \int_{0}^{\infty} d u^{2} \frac{\operatorname{Im} T_{V N}(u)}{u^{2}\left(u^{2}-\omega^{2}\right)} .
$$

The subtraction constants $l_{V}$ are fixed by the Thomson limit at $\omega=0$. Using eq.(66) we have $l_{\rho}=l_{\omega} / 9=-g^{2} /\left(4 M_{N}\right)$ and $l_{\phi}=0$. Evidently, processes with low thresholds dominantly influence the low energy region of the real and imaginary parts of $T_{V N}$. The convergence of the dispersion integral is slow, however, because $\operatorname{Im} T_{V N}$ increases linearly with energy. Therefore its high energy part also influences $\operatorname{Re} T_{V N}$. 
Our hadronic effective Lagrangian is expected to be valid up to a momentum and energy scale of order $1 \mathrm{GeV}$ and we expect formfactor suppressions at higher energies, in particular when the mesons are off-shell. Indeed, in the vacuum the VMD approach seizes to be valid at a scale $s=s_{V}$, and perturbative QCD takes over for $s>s_{V}$. We introduce formfactors for vector meson-baryon couplings:

$$
F_{V B}\left(k^{2}\right)=\frac{\Lambda_{V}^{2}-m_{V}^{2}}{\Lambda_{V}^{2}-k^{2}}
$$

where $k$ is the four momentum, $m_{V}$ the mass of the vector meson, and $\Lambda_{V}$ is the cutoff parameter. We choose $\Lambda_{V}=1.6 \mathrm{GeV}$, values close to those commonly used in Boson exchange $N N$ potentials. At the pseudovector pion-nucleon vertices we employ the empirical axial form factor

$$
G_{A}\left(k^{2}\right)=\frac{g_{A}}{\left(1-k^{2} / \Lambda_{A}^{2}\right)^{2}},
$$

with $\Lambda_{A} \simeq 1 \mathrm{GeV}$ [37] and assume that a slightly larger $\Lambda_{A}^{\Lambda, \Sigma}=1.2 \mathrm{GeV}$ applies for kaon couplings to baryons. We will discuss the sensitivity of the results to variations of the momentum scales $\Lambda$.

\subsection{The $\rho N$ amplitude}

For the $\rho$ meson the dominant contributions to the imaginary part of the scattering amplitude come from the inelastic processes $\rho N \rightarrow \pi N, \rho N \rightarrow \pi \Delta \rightarrow \pi \pi N$ and $\rho N \rightarrow$ $\omega N \rightarrow \pi \pi \pi N$. The corresponding one-loop diagrams are illustrated in Fig. 5. The propagating baryon in the loops of this figure can either be a nucleon or a $\Delta$ isobar. In the limit of large baryon mass only the diagrams, Figs. 5(a,c,e,f,m), survive. First we omit the form factor $G_{A}\left(k^{2}\right)$ and calculate the imaginary parts of the loops using the bare coupling $g_{A}$. For the $\pi N$ loops we get

$$
\operatorname{Im} T_{\rho N}^{(\pi N)}(\omega)=g_{A}^{2} \mathcal{H}\left(\omega, 0, m_{\pi}\right)
$$

where the function $\mathcal{H}$ is given (for $\omega>0$ ) by:

$$
\begin{aligned}
\mathcal{H}(\omega, \Delta, m)=\frac{g^{2}}{24 \pi f_{\pi}^{2}} & {\left[\frac { \sqrt { ( \omega - \Delta ) ^ { 2 } - m ^ { 2 } } } { ( \omega ^ { 2 } - 2 \omega \Delta ) ^ { 2 } } \left(3 \omega^{4}-4 \omega^{2} m^{2}+4 m^{4}+\Delta\left(8 \omega m^{2}-12 \omega^{3}\right)\right.\right.} \\
& \left.+\Delta^{2}\left(16 \omega^{2}-8 m^{2}\right)-8 \Delta^{3} \omega+4 \Delta^{4}\right) \\
& \left.-\frac{\Delta\left(\omega^{2}-4 m^{2}\right)^{\frac{3}{2}}}{2 \omega\left(\omega^{2}-4 \Delta^{2}\right)^{2}}\left(3 \omega^{2}-8 m^{2}-4 \Delta^{2}\right)\right]
\end{aligned}
$$

If we include diagram $5 \mathrm{k}$, which does not contribute to the imaginary part of $T_{\rho N}$, we can also evaluate the real part in the heavy baryon limit since all divergences cancel due to current conservation. We then find for the $\pi N$ loop contribution to $\operatorname{Re} T_{\rho N}$ in this limit :

$\operatorname{Re} T_{\rho N}^{(\pi N)}(\omega)=\frac{g^{2} g_{A}^{2}}{24 \pi f_{\pi}^{2}}\left[\frac{2 m_{\pi}^{3}}{\omega^{2}}-\frac{3 m_{\pi}}{2}+\left(3-\frac{4 m_{\pi}^{2}}{\omega^{2}}+\frac{4 m_{\pi}^{4}}{\omega^{4}}\right)\left(\sqrt{m_{\pi}^{2}-\omega^{2}} \Theta\left(m_{\pi}-\omega\right)-m_{\pi}\right)\right]$. 
This corresponds to the result obtained by using eq.(87) as input in the dispersion relation (84). Of course we have to take relativistic corrections and the axial form factor into account. Calculating the $\pi N$ loops fully relativistically and using $G_{A}\left(k^{2}\right)$ given in eq. (86) instead of $g_{A}$ we end up with $\operatorname{Im} T_{\rho N}^{(\pi N)}(\omega)$ as shown by the short-dashed line of Fig. 7a.

Note that for the $\pi N$ loops the function $\mathcal{H}\left(\omega, 0, m_{\pi}\right)$ greatly simplifies. The situation is more involved when we consider the $\pi \Delta$ loops. We find in the heavy baryon limit

$$
\operatorname{Im} T_{\rho N}^{(\pi \Delta)}(\omega)=2 g_{A}^{2} \mathcal{H}\left(\omega, M_{\Delta}-M_{N}, m_{\pi}\right) .
$$

Here $M_{\Delta}=1.232 \mathrm{GeV}$ is the $\Delta$-isobar mass. A fully relativistic calculation would require using the Rarita-Schwinger formalism which introduces ambiguities. Here we only take the axial form factor into account by replacing $g_{A}$ by a dipole $\pi N \Delta$ transition formfactor $G_{A}^{(\Delta)}\left(k^{2}\right)$ with $\Lambda_{A}^{(\Delta)}=\Lambda_{A}$. Adding this result to the $\pi N$ loops contribution gives rise to the long-dashed curve in Fig. 7a.

For the high energy part of the spectrum we have found the box diagram $5(\mathrm{~m})$ to be very important. Its fully relativistic evaluation gives

$$
\operatorname{Im} T_{\rho N}^{(\pi \omega)}(\omega)=g_{A}^{2} \mathcal{I}\left(\omega, m_{\omega}\right)
$$

where

$$
\mathcal{I}\left(\omega, m_{V}\right)=\frac{g_{\omega \rho \pi}^{2}}{48 \pi f_{\pi}^{4}} \frac{\omega^{2} \vec{k}^{3}\left[2 \vec{k}^{2} M_{N}^{2}-M_{N}\left(k_{0}-\omega\right)\left[\left(k_{0}-\omega\right)^{2}-\vec{k}^{2}\right]\right]}{M_{N}\left(M_{N}+\omega\right)\left(m_{\pi}^{2}-m_{V}^{2}+2 k_{0} \omega-\omega^{2}\right)^{2}}
$$

Here $g_{\omega \rho \pi}=1.2$ and we use $|\vec{k}|=\sqrt{\lambda\left(M_{N}+\omega, m_{V}, M_{N}\right)} /\left(2\left(M_{N}+\omega\right)\right)$ and $k_{0}=\sqrt{m_{V}^{2}+\vec{k}^{2}}$. The Källen function $\lambda$ is defined by

$$
\lambda(x, y, z)=\left(x^{2}-(y+z)^{2}\right)\left(x^{2}-(y-z)^{2}\right) .
$$

Including the axial formfactor and adding this to the contributions of the $\pi N$ and $\pi \Delta$ loops gives rise to the solid curve in Fig. 7a.

One might expect that processes $\rho N \rightarrow K \Sigma, K \Lambda$ also contribute to $T_{\rho N}$. We give the result from $K \Sigma$ and $K \Lambda$ loops in the heavy baryon limit, setting $m_{K}=m_{K^{+}}=m_{K^{0}}$ :

$$
\operatorname{Im} T_{\rho N}^{(K \Lambda, K \Sigma)}(\omega)=\frac{3}{8}(D-F)^{2} \mathcal{H}\left(\omega, M_{\Sigma}-M_{N}, m_{K}\right)+\frac{1}{24}(D+3 F)^{2} \mathcal{H}\left(\omega, M_{\Lambda}-M_{N}, m_{K}\right)
$$

Here $M_{\Lambda}=1.116 \mathrm{GeV}$ and $M_{\Sigma}=1.191 \mathrm{GeV}$ are the masses of $\Lambda$ and $\Sigma$ hyperons. Note that $\mathrm{SU}(3)$ factors reduce this amplitude already by about one order of magnitude with respect to the $\pi N$ loops, and a further reduction comes from the much smaller phase space and relativistic corrections. We can therefore safely neglect those contributions.

The real part of the $\rho N$ scattering amplitude is then determined by using $\operatorname{Im} T_{\rho N}$ (solid line of Fig. 7a) as input for the dispersion relation (84). The result is shown if Fig. 8a. The decomposition of $\operatorname{Re} T_{\rho N}$ into subprocesses is also given in this figure.

\subsection{The $\phi N$ amplitude}

For the $\phi$-meson the same contributions from the $K \Lambda$ and $K \Sigma$ loops as for the $\rho$-meson appear, but now larger by a factor of two:

$$
\operatorname{Im} T_{\phi N}(\omega)=\frac{3}{4}(D-F)^{2} \mathcal{H}\left(\omega, M_{\Sigma}-M_{N}, m_{K}\right)+\frac{1}{12}(D+3 F)^{2} \mathcal{H}\left(\omega, M_{\Lambda}-M_{N}, m_{K}\right)
$$


Note that from $\mathrm{SU}(3)$ symmetry the $\phi N$ amplitude is already four times smaller than the $\rho N$ amplitude with inclusion of $\pi N$ loops only. A further reduction comes from the smaller phase space because of the higher $K N$ threshold. Therefore the imaginary part and consequently also the real part (shown in Fig. 8c) of the $\phi N$ amplitude is much reduced as compared to the $\rho N$ case. This is evident by comparing Figs. 7a and c. The smallness of $\operatorname{Im} T_{\phi N}$ implies that effects of regularization by meson-baryon formfactors have little impact on the real part, at least in the region around the physical $\phi$ mass. We show results in Fig. 8c with inclusion of a kaon-baryon dipole formfactor using a cutoff $\Lambda_{A}^{(\Lambda, \Sigma)}=1.2 \mathrm{GeV}$. We mention however that diagrams such as Fig $5(1, \mathrm{~m})$, with $K^{*}$ and $K$ mesons exchanged in the box are not taken into account in the present calculation. Very little is known about such processes, but they may not be negligible.

\subsection{The $\omega N$ amplitude}

The Kroll-Ruderman term and the coupling to two pseudoscalar mesons do not exist for the $\omega$ meson. Consequently no such diagrams as in Figs. 5(a,b,c,e,f,h,i) contribute to the imaginary part of the $\omega N$ amplitude. The diagrams, Figs. $5(\mathrm{~d}, \mathrm{~g}, \mathrm{j})$ vanish in the heavy baryon limit. They contribute very little to the scattering amplitude even with the large coupling $g_{\omega}=17$. The dominant contributions come from processes where the $\omega$ turns into a $\pi \rho$ system which then interacts with the nucleon. The corresponding box diagrams, Fig. $5(\mathrm{~m})$ (with the role of $\rho$ and $\omega$ interchanged) give:

$$
\operatorname{Im} T_{\omega N}^{(1)}(\omega)=3 g_{A}^{2} \mathcal{I}\left(\omega, m_{\rho}\right),
$$

where $\mathcal{I}\left(\omega, m_{V}\right)$ is defined in eq. (92). At low energy the box diagram Fig. 5(1) becomes important since the rho mesons in the box interact strongly with the nucleons due to the large anomalous tensor coupling with $\kappa_{\rho}=6$. We find for this term:

$$
\operatorname{Im} T_{\omega N}^{(2)}(\omega)=\frac{g_{\omega \rho \pi}^{2} g^{2}\left(1+\kappa_{\rho}\right)^{2}}{128 \pi f_{\pi}^{2}} \frac{|\vec{l}|\left(\omega^{2}-m_{\pi}^{2}\right)^{2} \omega^{2}\left(4 M_{N}^{2}-m_{\pi}^{2}+4 M_{N} \omega+\omega^{2}\right)}{\left(M_{N}+\omega\right)^{4}\left(m_{\rho}^{2}-m_{\pi}^{2}-\omega^{2}+2 \omega l_{0}\right)^{2}} .
$$

using $|\vec{l}|=\sqrt{\lambda\left(M_{N}+\omega, m_{\pi}, M_{N}\right)} /\left(2\left(M_{N}+\omega\right)\right)$ and $l_{0}=\sqrt{m_{\pi}^{2}+\vec{l}^{2}}$ where $\lambda$ is the Källen function given in eq.(93).

Including also the contributions from vertex corrections in which the $\omega$ couples on one side directly to the nucleon and on the other side to the $\pi \rho$ system, we show the resulting imaginary part of the $\omega N$ amplitude in Fig. 7b replacing $g_{A}$ by the axial form factor and multiplying $\kappa_{\rho}$ by the monopole form factor (85). At low energies $\operatorname{Im} T_{\omega N}$ is small but rises strongly at higher energies. The high energy behaviour is sensitive to the meson-baryon form factors. This unavoidable model dependence also translates into $\operatorname{Re} T_{\omega N}$ (see Fig. 8b) when using the calculated $\operatorname{Im} T_{\omega N}$ in the dispersion relation (84). We should also point out, however, that the behaviour of $\operatorname{Im} T_{\omega N}$ can at least partly be checked against data. For example, the mechanisms that determine the long-dashed curve in Fig. $7 \mathrm{~b}$ also enter in the s-wave part of the $\pi N \rightarrow \omega N$ cross section. We find indeed satisfactory results for this cross section close to threshold. 


\subsection{Effective vector meson - nucleon scattering length}

In order to gain further insights into the basic features of the $V N$ amplitudes taken at $\vec{q}=0$, it is instructive to introduce complex effective scattering lengths,

$$
a_{V N}=\frac{M_{N}}{4 \pi\left(M_{N}+m_{V}\right)} T_{V N}\left(\omega=m_{V}\right),
$$

defined for "on-shell" vector mesons at rest. These scattering length parameters should of course be interpreted with care since the vector mesons themselves are unstable. Nevertheless the real and imaginary parts of $a_{V N}$ give a direct impression of the strong interaction shifts and widths that the vector mesons would experience in nuclear matter at low density.

The following effective scattering lengths are obtained for our standard scenario, with input specified in section 4 :

$$
\begin{aligned}
a_{\rho N} & =(0.04+i 1.62) \mathrm{fm}, \\
a_{\omega N} & =(3.34+i 2.1) \mathrm{fm}, \\
a_{\phi N} & =(-0.01+i 0.08) \mathrm{fm} .
\end{aligned}
$$

We observe that the $\rho N$ scattering length is strongly dominated by its imaginary part which describes the inelastic $\rho N \rightarrow \pi N, \pi \pi N$ etc. channels, whereas $\operatorname{Re} a_{\rho N}$ is persistently small, more than an order of magnitude smaller than $\operatorname{Im} a_{\rho N}$. In essence, this $a_{\rho N}$ causes a strong inelastic broadening, but almost no mass shift in matter. This is at striking variance with almost all previous analyses which have simply ignored the large imaginary part of $a_{\rho N}$.

The situation is quite different for the $\omega N$ scattering length. There one finds a large (attractive) real part of $a_{\omega N}$ which is mainly driven by the processes, Fig. 5(l,m). Thus the $\omega$ meson mass is indeed expected to be shifted downward in matter. At the same time one observes a substantial inelastic broadening.

Finally, the comparatively weak $\phi N$ interaction is reflected in the small $a_{\phi N}$, with almost vanishing real part and a moderate $\operatorname{Im} a_{\phi N}$ which comes primarily from the inelastic channels $\phi N \rightarrow K \Lambda, K \Sigma$.

The results, eqs.(99-101), are of course subject to some model dependence related to the form factors at the meson-baryon vertices that enter into loop diagrams, Fig. 5. Changes by $20 \%$ of the cutoffs $\Lambda$ in these form factors induce variations at the $30 \%$ level in the real and imaginary parts of $a_{V N}$. For example, increasing the cutoff $\Lambda_{A}^{(\Delta)}$ of the dipole $\pi N \Delta$ transition form factor from $1 \mathrm{GeV}$ to $1.3 \mathrm{GeV}$ will change the $\rho N$ scattering length to $a_{\rho N}=(-0.02+i 1.4) \mathrm{fm}$. It is thus uncertain whether the very small $\rho$ meson mass shift in matter is upward or downward, but the primary feature of a large $\operatorname{Im} a_{\rho N}$ remains in all cases. We have also examined corrections due to $\pi \pi$ rescattering in the basic $\rho N \rightarrow \pi \pi N$ box diagram (see Fig. 6) and found them to be small. Such mechanisms produce attraction, but their effects on the $\rho$ mass shift in matter are still weak (less than $5 \%$ at $\left.\rho=\rho_{0}\right)$ 


\section{$5 \quad$ Spectral densities and QCD sum rules in medium}

\subsection{In-medium spectral functions}

Combining our results for the $V N$ scattering amplitudes with the relations developed in chapter 3 we are now able to compute the in-medium correlation function $(72,74)$. We insert $T_{V N}$ into eq. (74) and display the spectral function in the form $-\left(12 \pi / \omega^{2}\right) \operatorname{Im} \Pi(\omega, \rho)$ for convenience, the one corresponding to eq.(8). This is done separately in each of the vector meson channels for different baryon densities $\rho$.

\subsubsection{Isovector spectral function}

In the isovector channel (see Fig. 9a) we observe the following characteristic features [25] as compared to the vacuum:

- The imaginary part of the in-medium rho meson self energy is strongly increased by the large $\operatorname{Im} T_{\rho N}$. This leads to a huge width in the $\mathrm{CC}$ correlation function, with the consequence that the rho meson resonance broadens and flattens.

- The spectrums starts now already at $\omega=m_{\pi}$, the threshold energy at which single pion production becomes possible. This generates a strong continuum in the correlation function below the $\rho$ resonance. We recall that the vacuum spectrum starts at a threshold $\omega=2 m_{\pi}$ coming from $\rho \rightarrow \pi^{+} \pi^{-}$decay.

- The shift of the $\rho$ meson pole position in matter is only marginal, given the small real part of the $\rho N$ scattering length. The in-medium rho meson mass is determined by

$$
m_{\rho}^{2}(\rho)=\stackrel{\mathrm{o}}{m}_{\rho}^{2}+\operatorname{Re}\left[\Pi_{\rho}^{\mathrm{vac}}\left(m_{\rho}^{2}\right)-\rho T_{\rho N}\left(m_{\rho}^{2}\right)\right] .
$$

With our standard scenario we find

$$
m_{\rho}^{2}(\rho)=m_{\rho}^{2}(0)\left(1-0.005 \frac{\rho}{\rho_{0}}\right)
$$

a very small shift at nuclear matter density, $\rho_{0}=0.17 \mathrm{fm}^{-3}$.

It is instructive to study the real part of the in-medium meson propagator,

$$
D_{\rho}(\omega, \vec{q}=0 ; \rho)=\frac{1}{\omega^{2}-\stackrel{\circ}{m}_{\rho}^{2}-\left(\Pi_{\rho}^{\operatorname{vac}}\left(\omega^{2}\right)-\rho T_{\rho N}(\omega)\right)} .
$$

The zero of $\operatorname{Re} D_{\rho}$ determines the in-medium mass. The result is shown in Fig. 10a. One clearly observes that, with rising density, the rho meson has little chance to survive as a quasi-particle. The large inelastic width in matter diffuses the well developed quasiparticle pole structure seen in the vacuum. The actual downward shift of strength in the spectrum in matter is caused entirely by the strong increase of the imaginary part of the in-medium self energy.

\subsubsection{Isoscalar spectral function}

For the $\omega$ meson the situation is different (see Fig. 9b). It starts out as a narrow resonance in vacuum. Both real and imaginary parts of $T_{\omega N}$ are large. The $\omega$ meson mass shifts 
downward and its width increases by an order of magnitude at $\rho=\rho_{0}$ as compared to the free width, but it survives as a quasi-particle in matter, at least up to densities $\rho \simeq \rho_{0}$. This is clearly seen in the real part of the in-medium $\omega$ meson propagator (see Fig. 10b), the zero of which moves downward with increasing density while the pronounced resonance pattern still persists.

\subsubsection{The $\phi$ spectral function}

Because of the relatively weak $\phi N$ interaction, the $\phi$ meson peak moves very little (see Fig. 9c). At $\rho=\rho_{0}$ its width increases by about one order of magnitude over its very small decay width in free space due to the opening of the inelastic channels $\phi N \rightarrow K \Lambda, K \Sigma$. The spectrum now start at the threshold for those processes, but the well developed $\phi$ resonance remains clearly visible at $\rho=\rho_{0}$.

\subsection{QCD sum rules in baryonic matter}

Our calculated in-medium CC correlation function at $\vec{q}=0$ is now used as input for the dispersion relation which represents the left side of the QCD sum rule at finite baryon density. We then examine the consistency with the operator product expansion, the right hand side of this sum rule.

As in the vacuum case described in section 2, it is useful to work with the quantities

$$
R^{(V)}(\omega ; \rho)=-\frac{12 \pi}{\omega^{2} g_{V}^{2}} \operatorname{Im} \Pi_{V}(\omega, \vec{q}=0, \rho) \Theta\left(s_{V}-\omega^{2}\right)+d_{V}\left(1+\frac{\alpha_{S}}{\pi}\right) \Theta\left(\omega^{2}-s_{V}\right)
$$

in each of the three vector meson channels $(V=\rho, \omega, \phi)$. The calculated spectrum $\operatorname{Im}_{V}$ (see eq.(76) ) is valid up to some (density dependent) scale $s_{V}(\rho)$ which still needs to be fixed. At higher energies beyond $s_{V}$ the perturbative QCD result takes over; here we assume that the coefficients $d_{V}$ do not change with density. After Borel transformation one obtains the in-medium QCD sum rules in a form analogous to eq.(36):

$$
\frac{1}{d_{V} \mathcal{M}^{2}}\left[12 \pi^{2} \frac{T_{V N}(0) \rho}{g_{V}^{2}}+\int_{0}^{\infty} d \omega^{2} R^{(V)}(\omega, \rho) e^{-\omega^{2} / \mathcal{M}^{2}}\right]=\tilde{c}_{0}+\frac{\tilde{c}_{1}}{\mathcal{M}^{2}}+\frac{\tilde{c}_{2}(\rho)}{\mathcal{M}^{4}}+\frac{\tilde{c}_{3}(\rho)}{2 \mathcal{M}^{6}}
$$

The first term on the left hand side corresponds to the Landau damping, the vector meson analogue of the Thomson scattering limit, with $T_{V N}(0)=g_{V}^{2} / 4 M_{N}$ for $V=\rho, \omega$. For the $\phi$ meson this term vanishes.

The coefficients $\tilde{c}_{1}, \tilde{c}_{2}$ and $\tilde{c}_{3}$ include the density dependence of condensates which have already appeared in the vacuum sum rule, as well as new condensates which only exist at finite baryon density. For the isovector and isoscalar channels we have [3]:

$$
\begin{aligned}
\tilde{c}_{0}^{\rho, \omega} & =c_{0}^{\rho, \omega}, \quad \tilde{c}_{1}^{\rho, \omega}=0 \\
\tilde{c}_{2}^{\rho, \omega}(\rho) & =c_{2}^{\rho, \omega}(\rho)+2 \pi^{2} A_{1}^{u+d} M_{N} \rho, \\
\tilde{c}_{3}^{\rho, \omega}(\rho) & =c_{3}^{\rho, \omega}(\rho)-\frac{10}{3} \pi^{2} A_{3}^{u+d} M_{N}^{3} \rho,
\end{aligned}
$$


where $c_{0}^{\rho, \omega}$ are the same as in the vacuum. For $c_{2}^{\rho, \omega}$ we use [3]:

$$
\begin{aligned}
c_{2}^{\rho, \omega}(\rho) & =\frac{\pi^{2}}{3}\left\langle\Omega\left|\frac{\alpha_{S}}{\pi} \mathcal{G}^{\mu \nu} \mathcal{G}_{\mu \nu}\right| \Omega\right\rangle \\
& \simeq \frac{\pi^{2}}{3}\left\langle 0\left|\frac{\alpha_{S}}{\pi} \mathcal{G}^{\mu \nu} \mathcal{G}_{\mu \nu}\right| 0\right\rangle-\frac{8 \pi^{2}}{27} M_{N}^{(0)} \rho
\end{aligned}
$$

where $M_{N}^{(0)}=750 \mathrm{MeV}$ is chosen for the nucleon mass in the chiral limit [38]. The contribution from the chiral condensate, $\left\langle\Omega\left|m_{u} \bar{u} u+m_{d} \bar{d} d\right| \Omega\right\rangle=\left\langle 0\left|m_{u} \bar{u} u+m_{d} \bar{d} d\right| 0\right\rangle+\rho \sigma_{N}$ with the nucleon sigma term $\sigma_{N} \simeq 45 \mathrm{MeV}$, is small and can be neglected.

The in-medium four-quark condensate determines $c_{3}^{\rho, \omega}(\rho)$. Its density dependence is not very well under control and requires some discussion. The basic question is to what extent ground state saturation, $\left\langle\Omega\left|(\bar{q} q)^{2}\right| \Omega\right\rangle \simeq\langle\Omega|\bar{q} q| \Omega\rangle^{2}$, is approximately valid in matter. This factorization of $\left\langle(\bar{q} q)^{2}\right\rangle$ holds in the mean field approximation where one neglects any excitations that can be reached by acting with $\bar{q} q$ on the ground state. In a nuclear medium as well as in the vacuum, scalar multi-pion excitations of the ground state are expected to be relevant, however. By analogy with eqs.(28) and (33) we parameterize:

$$
\left\langle\Omega(\rho)\left|\left(\bar{q} \gamma_{\mu} \gamma_{5} \lambda^{a} q\right)^{2}\right| \Omega(\rho)\right\rangle=-\left\langle\Omega(\rho)\left|\left(\bar{q} \gamma_{\mu} \lambda^{a} q\right)^{2}\right| \Omega(\rho)\right\rangle=\frac{16}{9} \kappa(\rho)\langle\Omega(\rho)|\bar{q} q| \Omega(\rho)\rangle^{2},
$$

where the density dependent factor $\kappa(\rho)$ now represents the in-medium deviation from simple factorization. Ground state saturation means $\kappa \equiv 1$. We now assume that the leading density dependence comes from the in-medium condensates $\langle\Omega(\rho)|\bar{q} q| \Omega(\rho)\rangle=$ $\langle 0|\bar{q} q| 0\rangle+\sigma_{N} \rho /\left(m_{u}+m_{d}\right)$, while the low-energy part of the spectrum of scalar excitations that enters in $\kappa(\rho)>1$ is approximately independent of $\rho$ (see also refs. [4, 26]). We therefore choose $\kappa(\rho) \equiv \kappa \simeq 2.36$ as in the vacuum and find

$$
c_{3}^{\rho, \omega}=-\frac{896}{81} \kappa \pi^{3} \alpha_{S}\left(\langle 0|\bar{q} q| 0\rangle^{2}-\frac{2 \sigma_{N} \rho}{m_{u}+m_{d}}\langle 0|\bar{q} q| 0\rangle\right)
$$

to leading order in density $\rho$. This may be a crude approximation which overestimates the density dependence. At least the tendency for $\left\langle(\bar{q} q)^{2}\right\rangle$ to decrease with increasing density is without question [27], since the difference between vector and axial vector current correlation functions, $\left|\left\langle\left|j_{V} j_{V}\right|\right\rangle-\left\langle\left|j_{A} j_{A}\right|\right\rangle\right|$, is proportional to $\langle\bar{q} q\rangle^{2}$ and tends to zero at the point of chiral restoration.

As pointed out in ref. [3], further in-medium contributions to eqs.(107-109) arise from matrix elements of the form $\left\langle N\left|\bar{q} \gamma_{\mu_{1}} D_{\mu_{2}} \ldots D_{\mu_{n+1}} q\right| N\right\rangle$ with gluon fields entering in the gauge covariant derivatives $D_{\mu}$. These matrix elements are proportional to the moments

$$
A_{n}^{q}=2 \int_{0}^{1} d x x^{n}[q(x)+\bar{q}(x)]
$$

of quark and antiquark distributions which can be determined, at some renormalization scale of order $1 \mathrm{GeV}$, from deep-inelastic lepton scattering on a nucleon. We use values as in ref.[3] and set $A_{1}^{u+d}=0.9, A_{3}^{u+d}=0.12$. Additional corrections of higher twist operators are smaller than the twist 2 contributions [28, 29] and discussed in ref. [19]. 
For the $\phi$ meson the coefficient $c_{2}^{\phi}$ is dominated by the strange quark condensate. Its in-medium change is given to leading order in density by

$$
\langle\Omega(\rho)|\bar{s} s| \Omega(\rho)\rangle=\langle 0|\bar{s} s| 0\rangle+y \frac{\rho \sigma_{N}}{m_{u}+m_{d}}
$$

with $y=\langle N|\bar{s} s| N\rangle /\langle N|\bar{u} u| N\rangle \simeq 0.2$. We then find

$$
\begin{aligned}
\tilde{c}_{0}^{\phi} & =c_{0}^{\phi}, \quad \tilde{c}_{1}^{\phi}=c_{1}^{\phi}, \\
\tilde{c}_{2}^{\phi}(\rho) & =c_{2}^{\phi}(\rho)+4 \pi^{2} A_{1}^{s} M_{N} \rho, \\
\tilde{c}_{3}^{\phi}(\rho) & =c_{3}^{\phi}(\rho)-\frac{20}{3} \pi^{2} A_{3}^{s} m_{N}^{3} \rho,
\end{aligned}
$$

where $c_{2}^{\phi}(\rho)$ and $c_{3}^{\phi}(\rho)$ are obtained from $c_{2,3}^{\phi}$ of eqs.(30-31) when replacing the vacuum $|0\rangle$ by $|\Omega(\rho)\rangle$. We use the values $A_{1}^{s}=0.05$ and $A_{3}^{s}=0.002$ of the strange quark moments as in ref. [3]. The resulting density dependent changes of the right hand (OPE) side of the QCD sum rule for the $\phi$ meson with respect to the vacuum are small.

In Figs. 11 we compare the "left hand side" with the "right hand side" of eq.(106) for the $\rho, \omega$ and $\phi$ channels at normal nuclear matter density $\rho=\rho_{0}=0.17 \mathrm{fm}^{-3}$, as a function of the Borel mass $\mathcal{M}$. For the energy scales $s_{V}$ in eq.(105) which separate the resonant parts of the spectrum from the high-energy QCD continuum, we use

$$
\begin{aligned}
& s_{\rho}=1.56 \mathrm{GeV}^{2}\left(1-0.1 \rho / \rho_{0}\right), \\
& s_{\omega}=1.65 \mathrm{GeV}^{2}\left(1-0.3 \rho / \rho_{0}\right), \\
& s_{\phi}=2.2 \mathrm{GeV}^{2}\left(1-0.01 \rho / \rho_{0}\right),
\end{aligned}
$$

in order to match the region of asymptotic Borel masses.

The overall consistency between calculated in-medium spectral functions and the density dependent operator product expansion is evidently quite remarkable. We should compare Figs. 11a-c with the corresponding vacuum sum rule analysis, Figs. 3a-c. Note that the difference between the $\rho=\rho_{0}$ and $\rho=0$ cases is large for the $\rho$ and $\omega$ meson channels, whereas it is marginal for the $\phi$ channel.

\subsection{Discussion}

\subsubsection{Comparison with previous work}

Hatsuda and Lee [3] have inserted the simplified parameterization

$$
R^{(V)}(\omega, \rho)=\mathcal{F}_{V}^{*} \delta\left(\omega^{2}-m_{V}^{* 2}(\rho)\right)+d_{V}\left(1+\frac{\alpha_{s}}{\pi}\right) \Theta\left(\omega^{2}-s_{V}^{*}(\rho)\right)
$$

in the "left hand side" of their in-medium QCD sum rule and fitted $\mathcal{F}_{V}^{*}, m_{V}^{*}$ and $s_{V}^{*}$. Apart from their choice of $\kappa=1$, their "right hand side" is the same as eq.(106). Their optimal fits gave

$$
\frac{m_{\rho, \omega}^{*}}{m_{\rho, \omega}}=1-(0.16 \pm 0.6) \frac{\rho}{\rho_{0}}
$$




$$
\begin{aligned}
\sqrt{\frac{s_{\rho, \omega}^{*}}{s_{\rho, \omega}}} & =1-(0.15 \pm 0.05) \frac{\rho}{\rho_{0}}, \\
\frac{\mathcal{F}_{\rho, \omega}^{*}}{\mathcal{F}_{\rho, \omega}} & =1-(0.24 \pm 0.07) \frac{\rho}{\rho_{0}} .
\end{aligned}
$$

Here $\mathcal{F}_{\rho}=12 \pi^{2} m_{\rho}^{2} / g_{\rho}^{2}$ is the vacuum pole strength for the $\rho$ meson, and an analogous expression holds for the $\omega$ meson. For the in-medium $\phi$ meson mass they find

$$
\frac{m_{\phi}^{*}}{m_{\phi}}=1-(0.15 \pm 0.5) y \frac{\rho}{\rho_{0}},
$$

with $y=0.1-0.2$. Jin and Leinweber [4] find relations consistent with eqs.(121-124). If we use $\kappa=2$ the results (121-124) change moderately within the errors indicated.

The fact that the in-medium masses $m_{\rho, \omega}^{*}$ both appear to decrease by (10-20\%) at $\rho=\rho_{0}$ in comparison with the vacuum masses $m_{\rho, \omega}$ has been interpreted as an indication of BR scaling [1]. In contrast, we find in our explicit calculation that the low-energy interaction of $\rho$ and $\omega$ mesons with surrounding matter differ strongly. The naive ansatz (120) remains reasonably justified in the isoscalar channel. There we have shown that, while the effective width of the $\omega$ meson at $\rho=\rho_{0}$ increases by about an order of magnitude over its (small) free width, the in-medium $\omega$ meson can still be identified as a quasi-particle with a well-defined effective mass that roughly resembles the fit, eq.(121). This is not the case, however, for the $\rho$ meson. The inelastic collision broadening in the isovector channel is so strong that it completely dominates the low-mass spectrum, whereas the pole mass of the rho meson at $\rho=\rho_{0}$ changes very little. The average spectral mass $\bar{m}$ in this channel, defined by

$$
\bar{m}^{2}(\rho)=\frac{\int_{0}^{1 \mathrm{GeV}^{2}} d \omega^{2} \omega^{2} R^{I=1}(\omega, \rho)}{\int_{0}^{1 \mathrm{GeV}^{2}} d \omega^{2} R^{I=1}(\omega, \rho)},
$$

drops roughly as $m_{\rho}^{*}$ in the parameterization (120), but this can obviously not be interpreted as a decreasing $\rho$ mass in matter, as we have pointed out by analyzing the zero of the real part of the rho meson propagator in Fig. 10a.

Asakawa and Ko [7] have used a more realistic $\rho$ meson spectral function than the schematic zero width form (120) and repeated the QCD Sum rule analysis with inclusion of a density dependent rho meson width. They have argued that a mass shift consistent with BR scaling still remains to be added. Our results for the $\rho$ meson do not support this conclusion.

The strong broadening of the $\rho$ meson spectral function in matter is also found by Chanfray et al. [36]. They include corrections of higher order in density induced by intermediate propagating pions. Our present calculations have focused on a consistent calculation of vector meson self-energies to leading order in density. We find that uncertainties in the (small) $\rho$ meson mass shift related to vertex form factors and high energy contributions to the spectral functions are in fact larger than the effects of higher order corrections in pion propagators. 


\subsubsection{Effective scattering amplitudes}

The main difference between the present approach and previous work is our explicit calculation of the complex vector meson-nucleon amplitudes at $\vec{q}=0$. In the low density limit the question about possible shifts of vector meson masses reduces to a statement about the corresponding effective $\rho N, \omega N$ and $\phi N$ scattering lengths. In previous discussions the large imaginary parts of these scattering lengths have simply been ignored. For example, Koike and Hayashigaki [22] deduce a real $\rho N$ scattering length $a_{\rho N} \simeq 0.45 \mathrm{fm}$, so that the relation $m_{\rho}^{* 2}-m_{\rho}^{2}=-4 \pi \rho\left(1+m_{\rho} / M_{N}\right) a_{\rho N}$ implies a downward mass shift of about $-45 \mathrm{MeV}$ at $\rho=\rho_{0}$. Our calculated effective scattering length (98) is qualitatively different. We find a large imaginary part $\operatorname{Im} a_{\rho N} \simeq 1 \mathrm{fm}$ which reflects the strong influence of the $\rho N \rightarrow \pi N, \pi \Delta$ inelastic channels. The real part $\operatorname{Re} a_{\rho N}$, on the other hand, is about one order of magnitude smaller than $\operatorname{Im} a_{\rho N}$. It may have different signs depending on details of cutoff procedures, but this is almost irrelevant in view of the large magnitude of the imaginary part.

Hatsuda, Lee and Shiomi [19] have pointed out that the low-density limit $\delta m_{V}^{2}=$ $-\rho T_{V N}$ is expected to break down already at a fraction of nuclear matter density $\rho_{0}$. This is indeed the case, given that the magnitude of $T_{V N}$ is large for $V=\rho, \omega$. It is necessary to use the full vector meson propagator $(\overline{73})$, with the self-energy $\Pi_{V}=-\rho T_{V N}$ iterated to all orders, and this has in fact been done when deriving the spectral function (76). Additional higher order corrections in $\Pi_{V}$ as calculated e.g. in ref. 36], do not lead to substantial changes for $\rho \lesssim \rho_{0}$.

\section{$6 \quad$ Summary and concluding remarks}

(1) Guided by the chiral meson-baryon effective Lagrangian with inclusion of vector mesons and electromagnetic interactions, we have calculated the spectral distributions of current-current correlation functions in each of the $\rho-, \omega$-, and $\phi$-meson channels, both in the vacuum and in baryonic matter. We observe perfect consistency with QCD sum rules in all cases.

(2) Our results do not suggest a universal scaling law for in-medium vector meson masses. We find almost no mass shifts for $\rho$ and $\phi$-mesons in matter, whereas the $\omega$ meson experiences a substantial (attractive) shift.

(3) The $\rho N$ and $\omega N$ effective scattering amplitudes are governed by large inelasticities. For the $\rho$ meson channel the inelastic broadening moves a large amount of the spectral strength down to the continuum below the free $\rho$ meson resonance. This broadening is so strong that the $\rho$ meson is unlikely to survive as a quasiparticle at densities much above $\rho_{0}$, the density of normal nuclear matter. In contrast, the $\omega$ meson, with its small width in vacuum to start with, can still be identified as a quasiparticle in matter at $\rho=\rho_{0}$. The $\phi N$ interaction is altogether weak. Consequently, the $\phi$ meson is expected to have almost no mass shift. Although its width at $\rho=\rho_{0}$ increases by about an order of magnitude over its free width, it still persists as a relatively narrow resonance. 
(4) To the extent that the changes of vector meson spectra in matter are driven by an effective chiral Lagrangian, they represent the dynamics and symmetry breaking pattern characteristic of low energy QCD. These features enter at the same time in the operator product expansion part of the QCD sum rules. Whether they directly reflect a tendency toward chiral symmetry restoration at high density is less obvious.

(5) Our calculations indicate that the lifetime of the $\omega$ meson in matter at $\rho=\rho_{0}$ decreases to about $\tau_{\omega} \sim 1.5 \mathrm{fm} / \mathrm{c}$. The production of "slow" $\omega$ mesons in a nuclear environment can therefore make in-medium changes of the $\omega$ meson spectrum visible. Such processes can be explored with the upcoming HADES lepton pair spectrometer at GSI. A reaction of particular interest is $\pi^{-} p \rightarrow \omega n$ in heavy nuclei with subsequent decay $\omega \rightarrow e^{+} e^{-}$and a kinematic cut focusing on low $\omega$ meson momenta [39, 40].

(6) For the lifetime of the $\phi$ meson we still expect $\tau_{\phi} \sim 10 \mathrm{fm} / \mathrm{c}$ at $\rho=\rho_{0}$ which exceeds nuclear dimensions. We mention that once the effective in-medium $K^{+}$and $K^{-}$ masses are introduced according to ref. [41], a slight downward shift of the $\phi$ meson is expected (by 1-2\% at $\rho=\rho_{0}$ ), and its lifetime decreases to about $6 \mathrm{fm} / \mathrm{c}$.

Acknowledgments:

We are grateful to Gerry Brown, Bengt Friman, Mannque Rho and Madeleine Soyeur for stimulating discussions.

\section{References}

[1] G.E. Brown and M. Rho, Phys. Rev. Lett. 66 (1991) 2720.

[2] K. Saito, K. Tsushima and A.W. Thomas, Nucl Phys. A 609 (1996) 339.

[3] T. Hatsuda and S.H. Lee, Phys. Rev. C 46 (1992) R34.

[4] X. Jin and D.B. Leinweber, Phys. Rev. C 52 (1995) 3344.

[5] M. Herrmann, B.L. Friman and W. Nörenberg, Nucl. Phys. A 560 (1993) 411.

[6] G. Chanfray and P. Schuck, Nucl. Phys. A 555 (1993) 329.

[7] M. Asakawa and C. M. Ko, Phys. Rev. C 48 (1993) 526.

[8] G.Q. Li, C.M. Ko and G.E. Brown, Phys. Rev. Lett. 75 (1995) 4007.

[9] W. Cassing, W. Ehehalt and C.M. Ko, Phys. Lett. B 363 (1995) 35.

[10] G. Agakichiev et al., Phys. Rev. Lett. 75 (1995) 1272.

[11] M. Masera et al., Nucl. Phys. A 590 (1995) 93c.

[12] H.C. Jean, J. Piekarewicz and A.G. Williams, Phys. Rev. C 49 (1994) 1981 
[13] E.V. Shuryak, Rev. Mod. Phys. 65 (1993) 1

[14] F. Klingl, N. Kaiser and W. Weise, Z. Phys. A 356(1996)193.

[15] V.A. Novikov, M.A. Shifman, A.I. Vainshtein and V.I. Zakharov, Fortschr. Phys. 32 (1984) 585.

[16] L.J. Reinders, H. Rubinstein and S. Yazaki, Phys. Reports127 (1985) 1.

[17] M.A. Shifman, A.I. Vainshtein and V.I. Zakharov, Nucl. Phys. B 147 (1979) 385.

[18] V.A. Novikov, M.A. Shifman, A.I. Vainshtein, M.B. Voloshin and V.I. Zakharov, Nucl. Phys. B 237 (1984) 525.

[19] T. Hatsuda, S.H. Lee and H. Shiomi, Phys. Rev. C 52 (1995) 3364;

[20] V. Gimenez, J. Bordes and J. Penarrocha, Nucl. Phys. B 357 (1991) 3.

[21] C.A. Dominguez and J. Sola, Z. Phys. C 40 (1988) 63; R. A. Bertlmann et al., Z. Phys. C 39 (1988) 231; G. Launer, S. Narison and R. Tarrach, Z. Phys. C 26 (1984) 433 ;

[22] Y. Koike and A. Hayashigaki, preprint: nucl-th/9609001, Aug 96

[23] E.G. Drukarev and E.M. Levin, Nucl. Phys. A 511 (1990) 679

[24] N. Kaiser, P.B Siegel and W. Weise, Nucl. Phys. A 594 (1995) 325; N. Kaiser, T. Waas and W.Weise, Nucl. Phys. A 612 (1997) 297.

[25] F.Klingl and W.Weise, Nucl. Phys. A 606 (1996) 329.

[26] D.B. Leinweber, Ann. Phys., 254 (1997) 328.

[27] S.H. Lee talk at the International Workshop on Hadrons in Dense Matter, GSI, Darmstadt, July 3-5 (1996), and private communication.

[28] S. Choi, T. Hatsuda, Y. Koike and S. H. Lee, Phys. Lett. B 312 (1993) 351.

[29] S. H. Lee, Phys. Rev. D49 (1994) 2242.

[30] L.M. Barkov et al., Nucl. Phys. B 256 (1985) 365.

[31] S.I. Dolinsky et al., Phys. Reports 202 (1991) 99.

[32] L.M. Barkov et al., JETP Lett. 46 (1987) 164.

[33] P.M. Ivanov et al., Phys. Lett. B 107 (1981) 297.

[34] F. Mane et al., Phys. Lett. B 112 (1982) 178.

[35] A. Cordier et al., Phys. Lett. B 110 (1982) 335. 
[36] G. Chanfray, R. Rapp and J. Wambach, Phys. Rev. Lett. 76 (1996) 368.

[37] T. Kitagaki et al., Phys. Rev. D 28 (1983) 436.

[38] B. Borasoy and U.-G. Meissner, Phys. Lett. B 365 (1996) 285.

[39] W. Schön, H. Bokemeyer, W. Koenig and V. Metag, Acta Phys. Polonica B 27 (1996) 2959.

[40] W. Cassing, Y.S. Golubeva, A.S. Iljinov and L.A. Kondratyuk, Phys. Lett. B 396 (1997) 26.

[41] T. Waas, N. Kaiser and W. Weise, Phys. Lett. B 379 (1996) 34; T. Waas, M. Rho and W.Weise, Nucl. Phys. A (1997), in print.

[42] T. Ericson and W.Weise, Pions and Nuclei, Clarendon Press, Oxford 1988. 


\section{Figure Captions:}

Figure 1: The ratio $\sigma\left(e^{+} e^{-} \rightarrow\right.$ hadrons $) / \sigma\left(e^{+} e^{-} \rightarrow \mu^{+} \mu^{-}\right)$as a function of the total c.m. energy. The isovector, isoscalar and the $\phi$ meson channels, corresponding to the currents (3-5), are shown separately in Figs. 1a, b and c, respectively. The data are taken from refs. [30-35]. In the $\phi$ channel the high energy data sample summarizes $K \bar{K}+n \pi$ final states. The solid lines are the results of the VMD model. The dashed lines represent the perturbative QCD limit.

Figure 2: Diagrammatic representation of the full photon propagator (wavy line) and of the full vector meson (V) propagator.

Figure 3: Comparison of the Borel transformed right side (operator product expansion, OPE) and left side (dispersion relation, DR) of the QCD sum rules for the isovector $(\mathrm{I}=1)$, isoscalar $(\mathrm{I}=0)$ and $\phi$ meson channels, as a function of the Borel mass $\mathcal{M}$ (see eq.(36)). The solid line is the result using our spectral density which describes the experimental data. The short dashed line is the simplified ansatz using a delta function for each vector meson resonance.

Figure 4: Feynman rules for the vertex functions derived from the effective Lagrangian of the coupled system of vector mesons, pseudoscalar mesons and baryons. Examples are shown here for interactions involving $\rho$ mesons and pions (including the $\pi \rho \omega$ coupling). Solid lines: nucleons; double solid lines: $\Delta$-isobars; dashed lines: pions; vector mesons are drawn as "zig zag" lines. The generalization to flavour $\mathrm{SU}(3)$ is straightforward. Here $S$ and $T$ are the $\Delta \rightarrow N$ spin and isospin transition operators and $\vec{\Theta}$ is the isospin operator for the $\Delta$ (see ref. 42 ).

Figure 5: Processes contributing to the $\rho N$ scattering amplitude. The dashed lines represents pions, the wavy line describes the $\rho$ meson. The incoming baryon (solid line) is always a nucleon while the intermediate baryon can be either a nucleon or a delta.

Figure 6: Two-loop contribution to the $\rho N$ scattering amplitude including $\pi \pi$ interactions.

Figure 7: Imaginary parts of the $V N$ scattering amplitudes in the isovector (a), isoscalar (b) and $\phi$ meson (c) channels. Solid lines: total results. Dashed lines show decomposition into selected subchannels.

Figure 8: Real and imaginary parts of $V N$ scattering amplitudes in different channels. Solid lines: imaginary parts used as input for dispersion relations to generate the real parts shown as long-dashed lines. Fig. 8a also shows separate contribution from $\rho N \rightarrow \pi N$ (with $\left.g_{A}=1\right)$ and $\rho N \rightarrow \pi N+\pi \Delta$ (with full axial formfactor).

Figure 9: Spectra of the current-current correlation functions in different channels, shown at zero (dashed lines), half (long dashed) and normal nuclear density (solid lines). 
Figure 10: Real part of in-medium vector meson propagator. The lines correspond to zero (dashed), half (long dashed) and normal nuclear density (solid).

Figure 11: The Borel transformed "left side" (l.s.) (from dispersion relation, dot dashed) and "right side" (r.s.) (from OPE, solid line) of the QCD sum rule (see eq.(106)) at normal nuclear density. For comparison we also show the vacuum results of Fig. 3. 

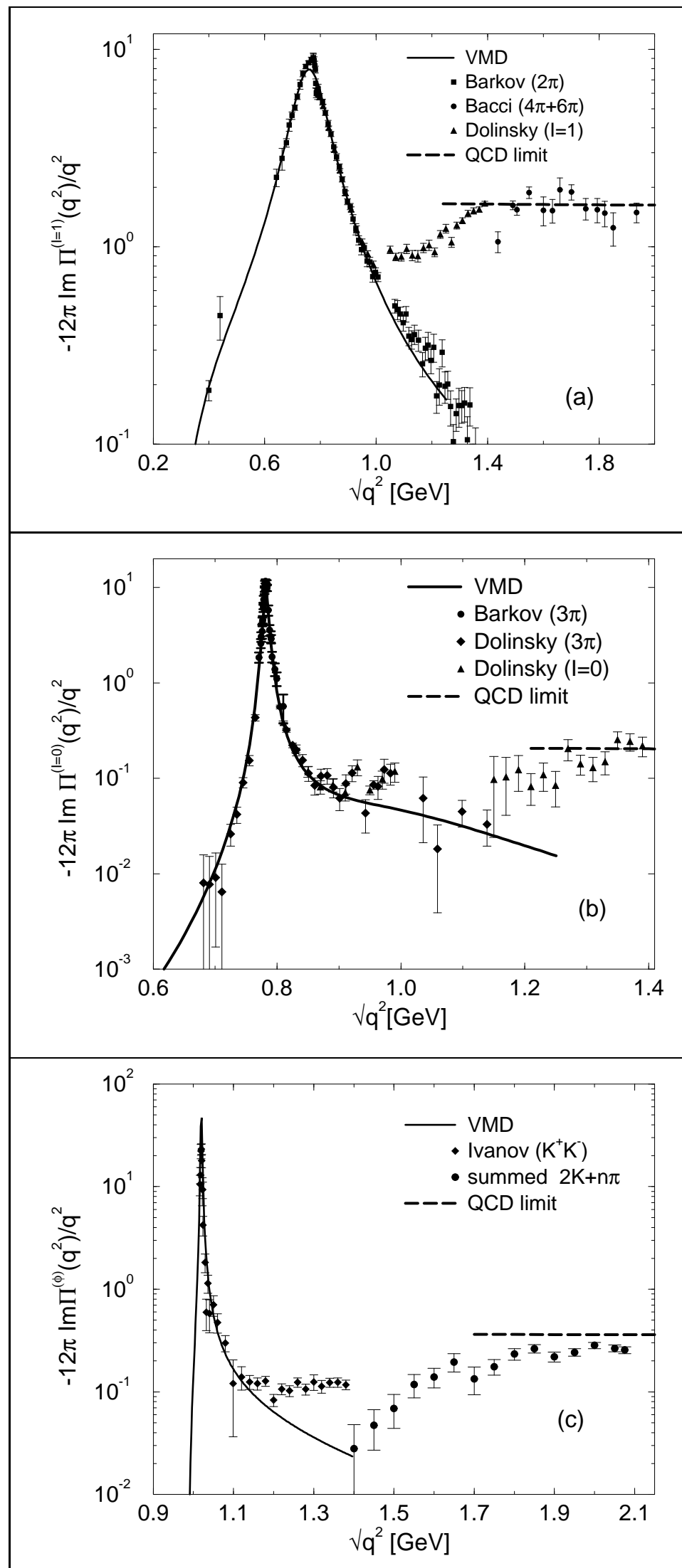

Fig.1 


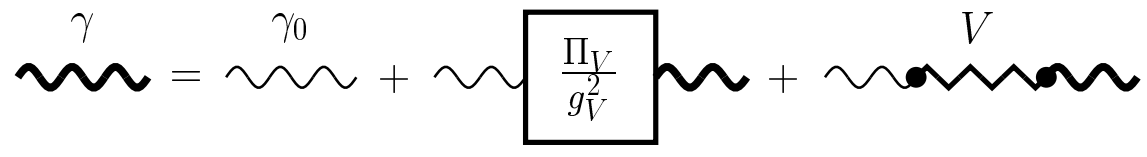

$$
\begin{aligned}
& \text { ram } \frac{\Pi_{V}}{g_{V}} h+\sim \frac{\Pi_{V}}{g_{V}} \text { wan }
\end{aligned}
$$

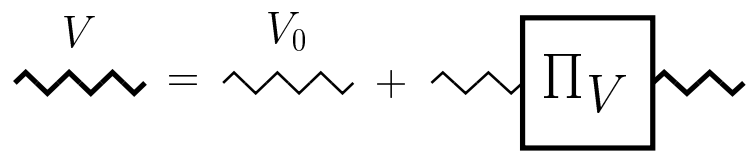



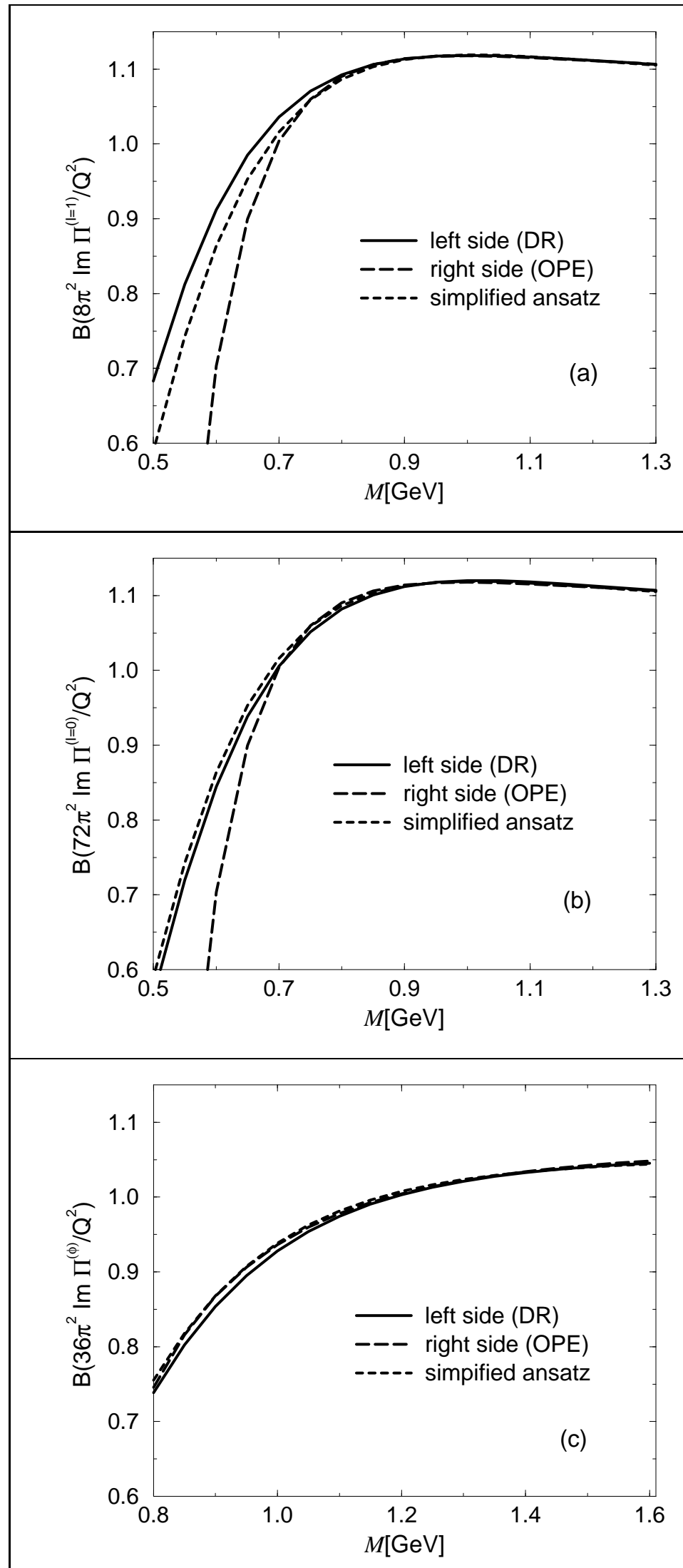

Fig.3 

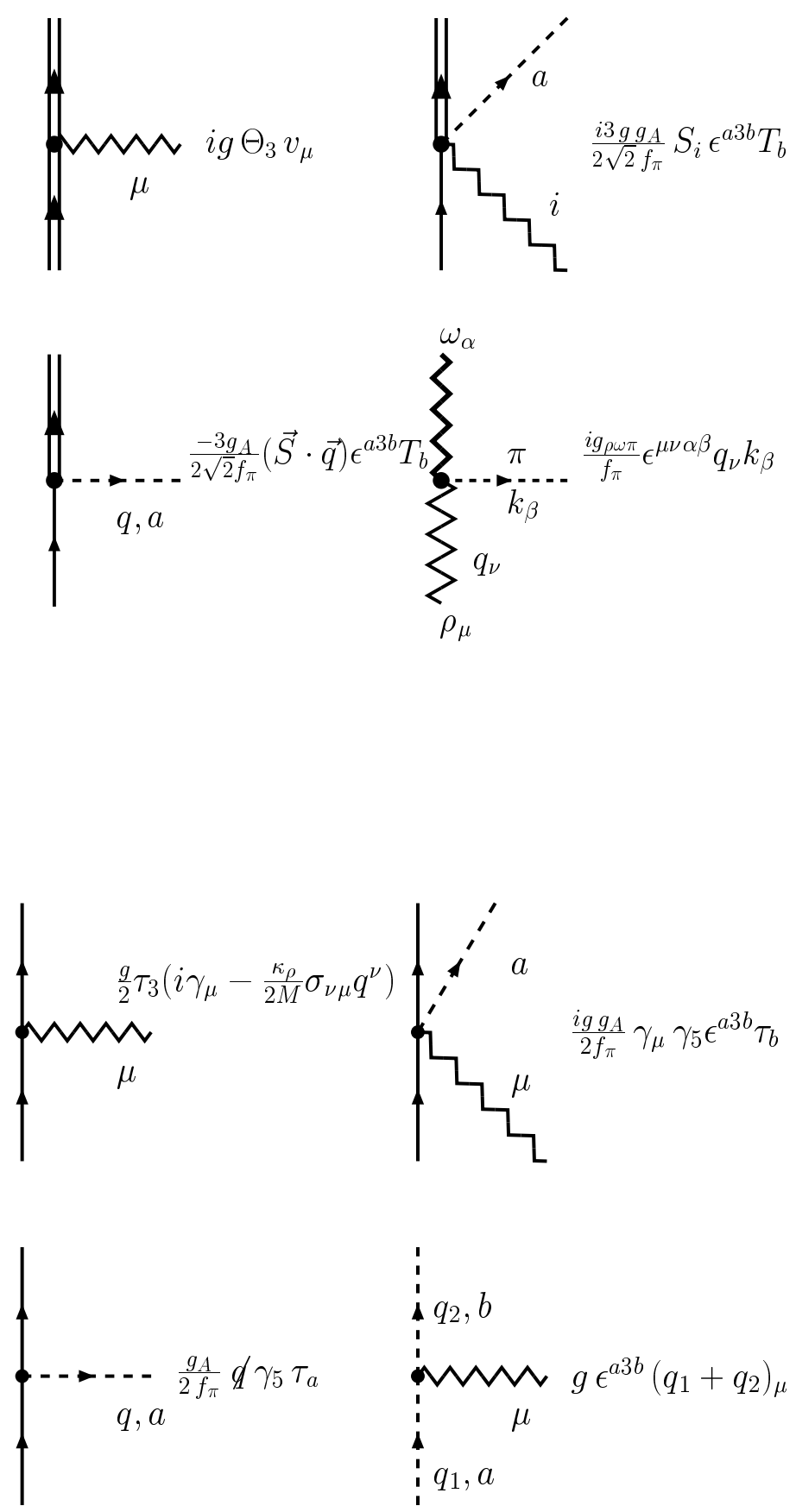

Fig.4 
:-
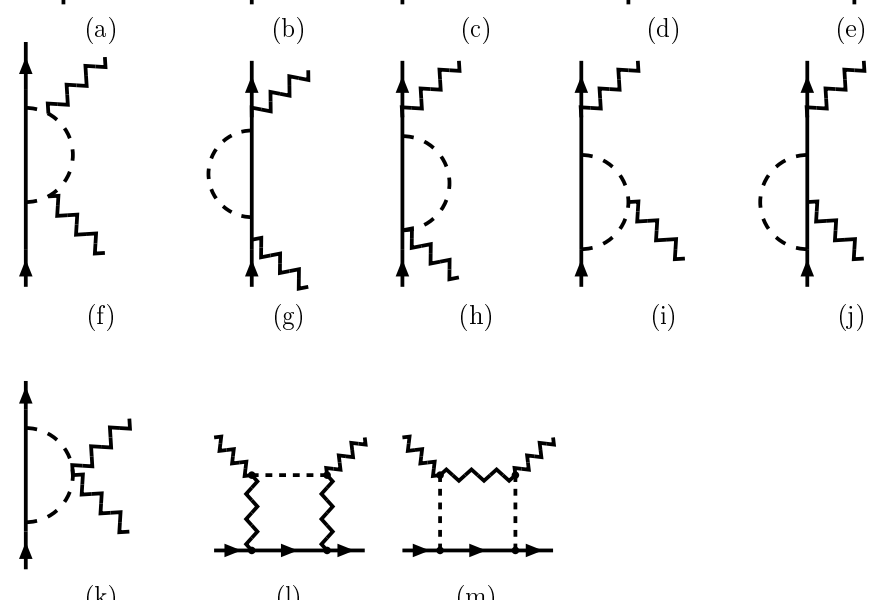

(k)

(1)

(m)

Fig.5

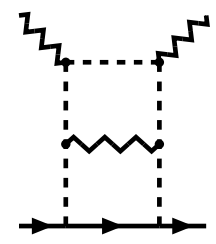

Fig.6 

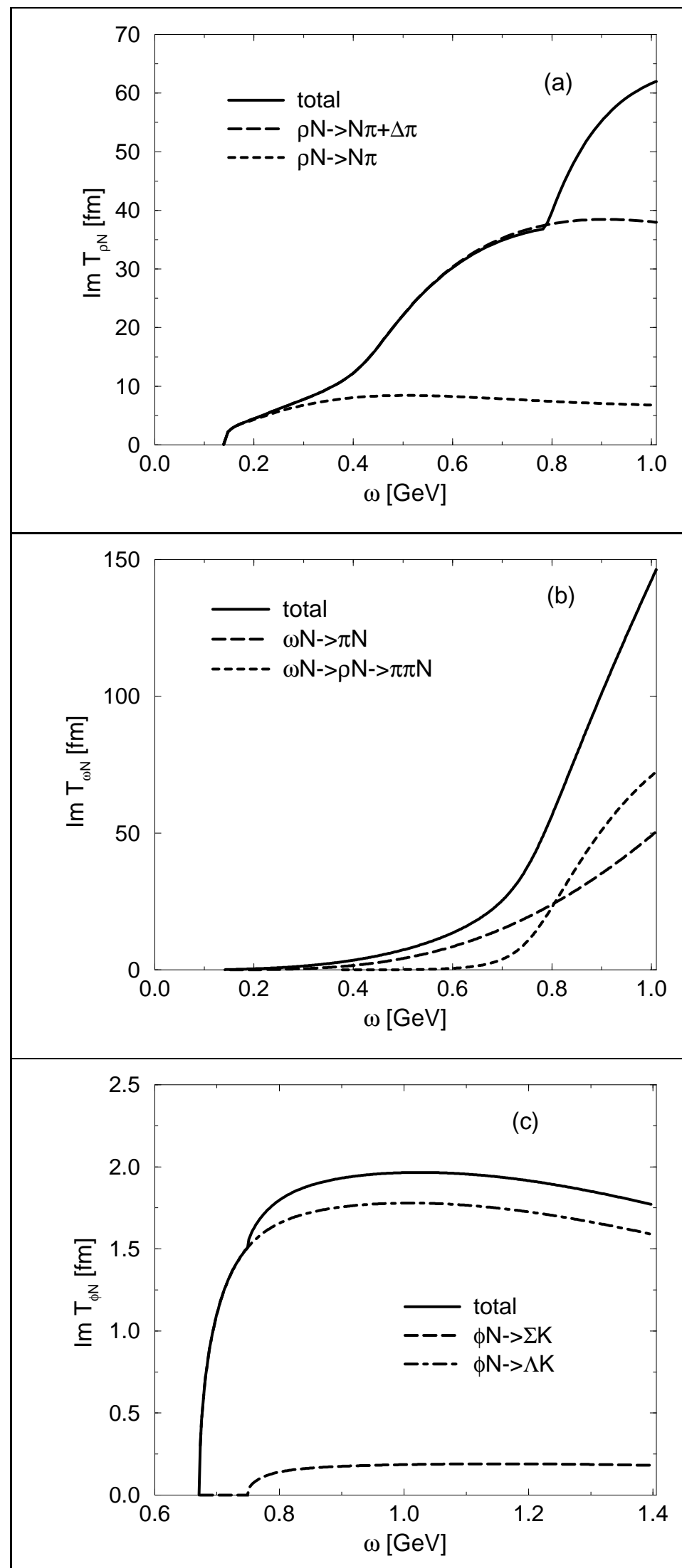

Fig.7 

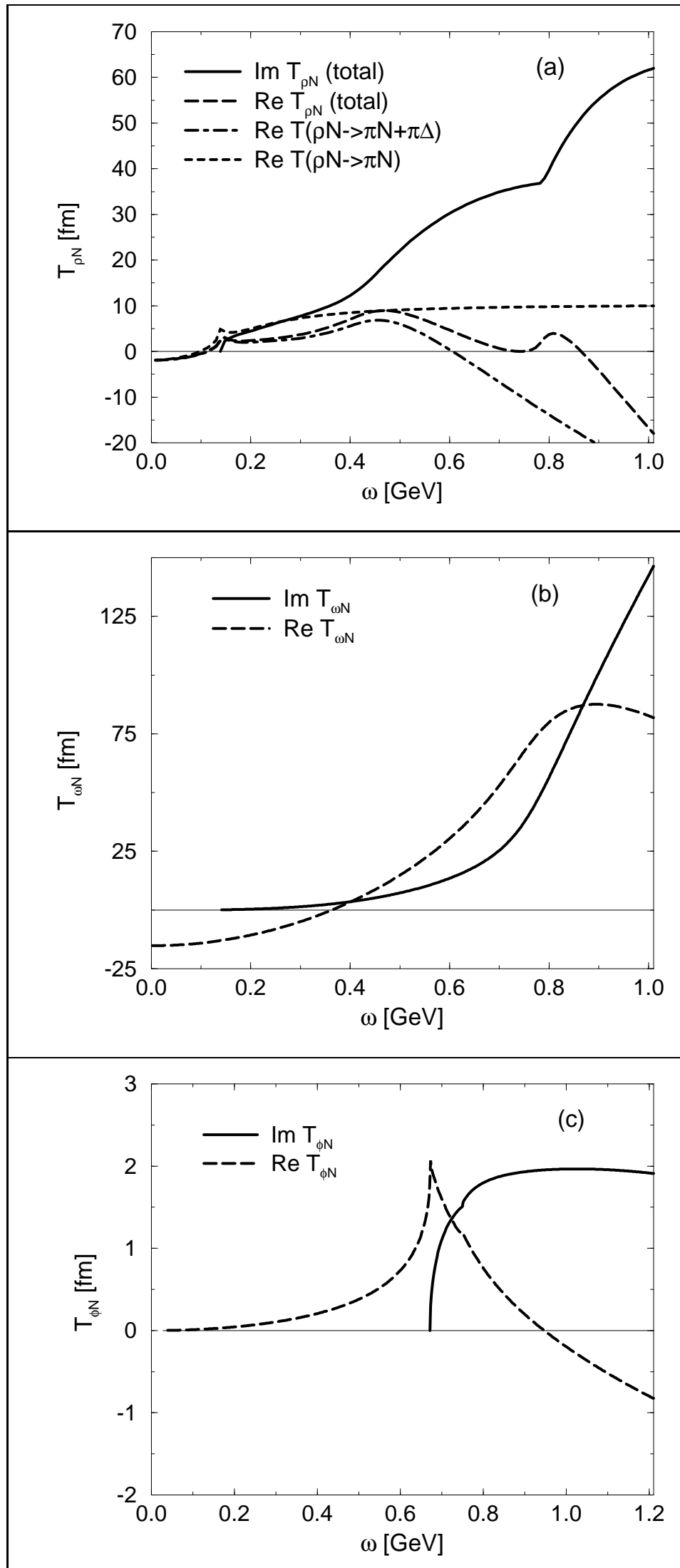

Fig.8 

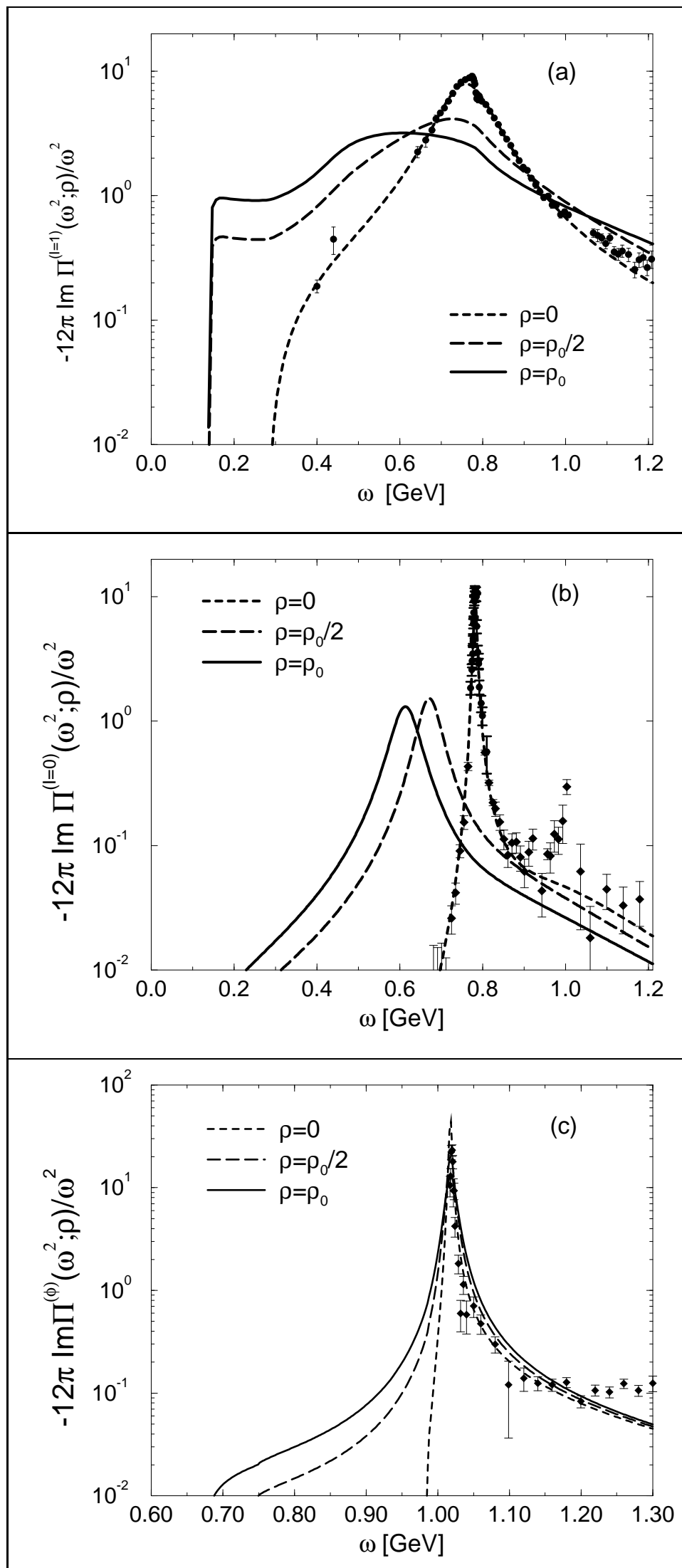

Fig.9 

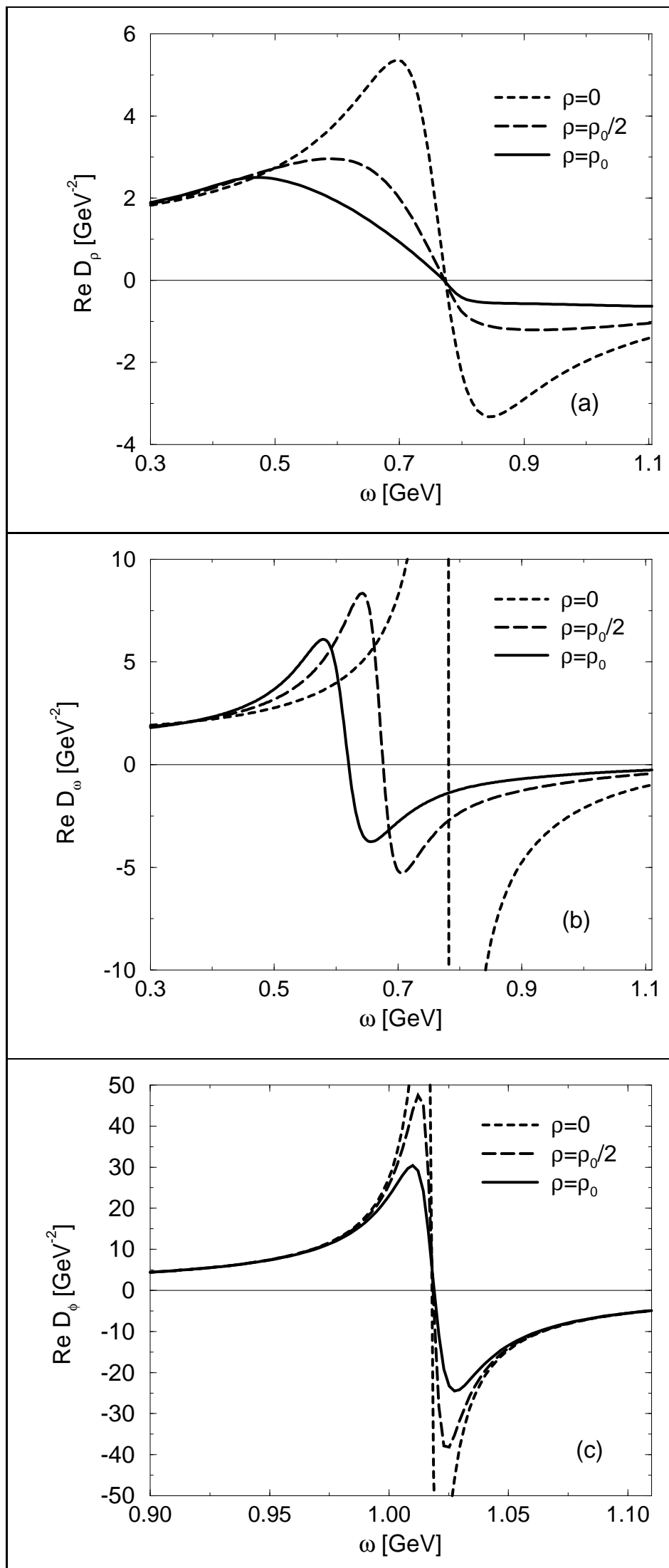

Fig. 10 

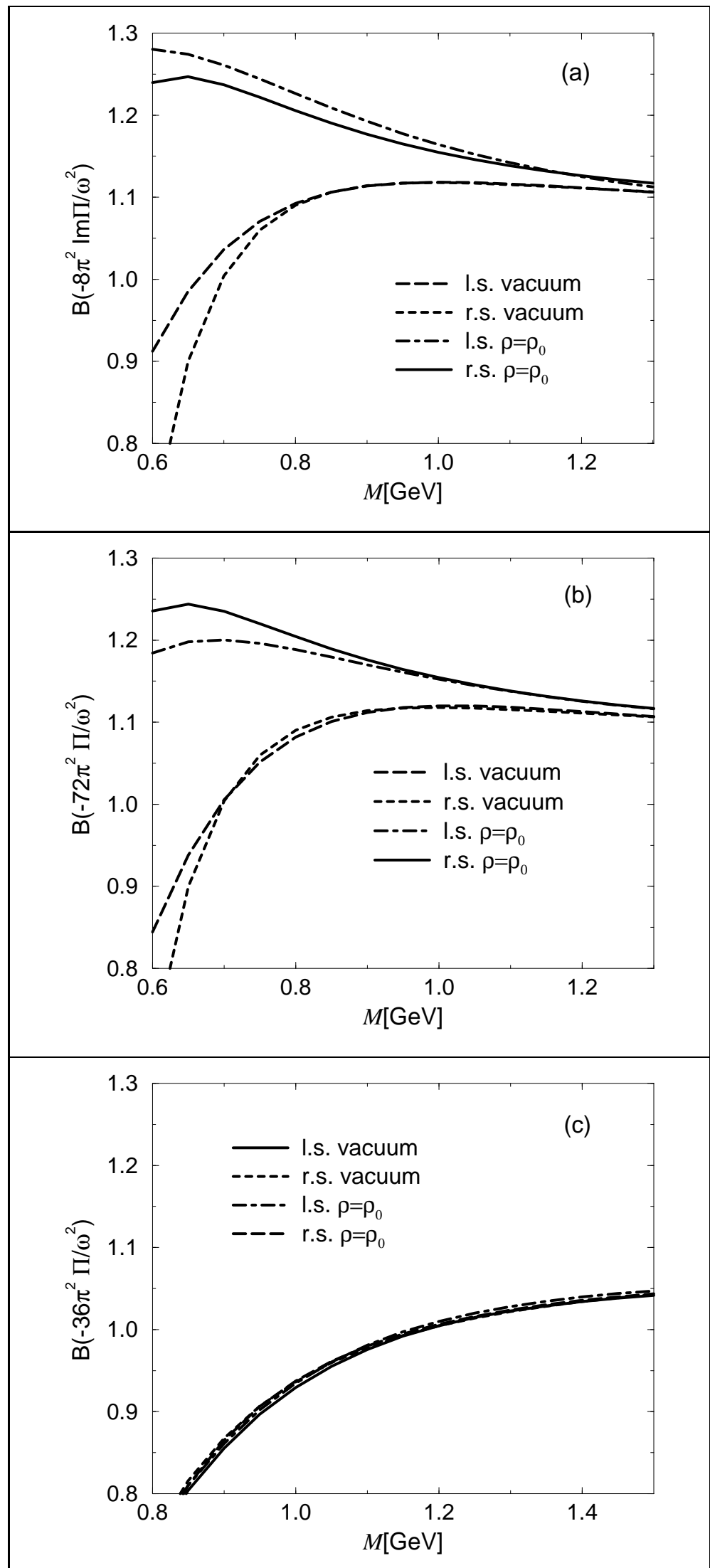

Fig.11 